Archives for Mathematical Logic manuscript No.

(will be inserted by the editor)

\title{
Saturated models in institutions
}

\author{
Răzvan Diaconescu · Marius Petria
}

the date of receipt and acceptance should be inserted later

Keywords Saturated models · institutions · institution-independent model theory

Abstract Saturated models constitute one of the powerful methods of conventional model theory, with many applications. Here we develop a categorical abstract model theoretic approach to saturated models within the theory of institutions. The most important consequence is that the method of saturated models becomes thus available to a multitude of logical systems from logic or from computing science.

In this paper we define the concept of saturated model at an abstract institution-independent level and develop the fundamental existence and uniqueness theorems. As an application we prove a general institution-independent version of the Keisler-Shelah isomorphism theorem "any two elementarily equivalent models have isomorphic ultrapowers" (assuming Generalized Continuum Hypothesis).

\section{Introduction}

1.1 Institution-independent Model Theory

The theory of "institutions" [21] is a categorical abstract model theory which formalizes the intuitive notion of logical system, including syntax, semantics, and the satisfaction between them. It provides the most complete form of abstract model theory, the only one including signature morphisms, model reducts, and even mappings (morphisms) between logics as primary concepts. Institution have been recently also extended towards proof theory $[36,15]$ in the spirit of categorical logic [28].

The concept of institution arose within computing science (algebraic specification) in response to the population explosion among logics in use there, with the ambition of doing as much as possible at a level of abstraction independent of commitment to any particular logic $[21,38,19]$. Besides its extensive use in specification theory (it has become the most fundamental mathematical structure in algebraic specification theory), there have been several substantial developments towards an "institution-independent" (abstract) model theory [42,43, 11, 13, 12, 26, 25, 37,9, 24, 35]. A monography dedicated to this topic is [17] and [14] is a relatively recent survey.

The significance of institution-independent model theory is manifold. First, it provides model theoretic results and analysis for various logics in a generic and uniform way. Apart of reformulation of standard concepts and results in a very general setting, thus applicable to many logical systems,

Institute of Mathematics "Simion Stoilow" of the Romanian Academy E-mail: Razvan.Diaconescu@imar.ro · Şcoala Normală Superioară Bucureşti E-mail: mariuspyah@yahoo.com 
some of them very different from the conventional logics, institution-independent model theory has already produced a series of new significant results in conventional model theory [13,26,37,9,24].

Institution-independent model theory provides a new top-down way of doing model theory, making explicit the generality and power of concepts by placing them at the right level of abstraction and thus extracting the essence of the results independently of the largely irrelevant details of the particular logic in use. This leads to a deeper conceptual understanding guided by a structurally clean causality. Concepts come naturally as presumed features that "a logic" might exhibit or not, hypotheses are kept as general as possible and introduced on a by-need basis, results and proofs are modular and easy to track down despite their sometimes very deep content.

\subsection{Summary and Contributions of this Work}

Saturated models constitute one of the powerful methods of conventional model theory, with many applications. For example this can be seen clearly in the classic textbook [6]. In this paper we define the concept of saturated model in arbitrary institutions and develop the fundamental results for an institution-independent saturated model theory. This makes available the method of saturated models to a large variety of logics from computing science and logic.

Our first fundamental result is an existence theorem showing that (under certain conditions for the institution) each model can be 'embedded elementarily' into a saturated model. Another fundamental result is a uniqueness theorem for the saturated models which are 'sufficiently small'. Both these properties, crucial in the applications, generalize corresponding first order model theory results of [33] (Lem. 5.1.4 and Thm. 5.1.13 in [6]). In the last section we develop an institution-independent version of a well known result in conventional concrete model theory showing that under certain conditions, ultraproducts of models are saturated, which leads to an institution-independent proof of the famous Keisler-Shelah isomorphism theorem "any two elementarily equivalent models have isomorphic ultrapowers". This proof has the merit that it separates clearly the ultrafilter part from the proper model theoretic part, the latter being shown to be institution-independent. One thus obtains a general version of Keisler-Shelah isomorphism theorem, under the Generalized Continuum Hypothesis, which can be applied to a variety of logics formalized as institutions.

Our general institution-independent concepts are illustrated by the classical first order model theory framework but also by the less conventional logics of partial algebra and preordered algebra. Applications to many other logics are of course expected.

The paper is organized as follows. The first technical section introduces the institution theoretic preliminaries and recalls necessary fundamental concepts of institution-independent model theory such as model amalgamation, elementary diagrams, internal logic, and finitary, small, quasirepresentable signature morphisms. The next section introduces the institution-independent concept of saturated model and proves the existence theorem. The third technical section develops the uniqueness property of saturated models. The last technical section is devoted to the institutionindependent generalization of Keisler-Shelah isomorphism theorem.

\section{Institution-independent Model Theoretic Preliminaries}

\subsection{Categories}

We assume the reader is familiar with basic notions and standard notations from category theory; e.g., see [30] for an introduction to this subject. Here we recall very briefly some of them. By way of notation, $|\mathbb{C}|$ denotes the class of objects of a category $\mathbb{C}, \mathbb{C}(A, B)$ the set of arrows with domain $A$ and codomain $B$, and composition is denoted by “;” and in diagrammatic order. The category of sets 
(as objects) and functions (as arrows) is denoted by $\mathbb{S e t}$, and $\mathbb{C A} \mathbb{T}$ is the category of all categories. ${ }^{1}$ The opposite of a category $\mathbb{C}$ (obtained by reversing the arrows of $\mathbb{C}$ ) is denoted $\mathbb{C}$. .

Given a functor $\mathcal{U}: \mathbb{C}^{\prime} \rightarrow \mathbb{C}$, for any object $A \in|\mathbb{C}|$, the comma category $A / \mathcal{U}$ has arrows $f: A \rightarrow \mathcal{U}(B)$ as objects (sometimes denoted as $(f, B))$ and $h \in \mathbb{C}^{\prime}\left(B, B^{\prime}\right)$ with $f ; \mathcal{U}(h)=f^{\prime}$ as arrows $(f, B) \rightarrow\left(f^{\prime}, B^{\prime}\right)$.

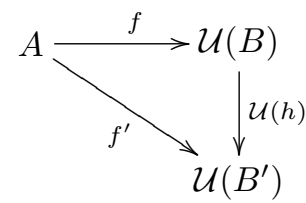

When $\mathbb{C}=\mathbb{C}^{\prime}$ and $\mathcal{U}$ is the identity functor the category $A / \mathcal{U}$ is denoted by $A / \mathbb{C}$.

A $J$-(co)limit in a category $\mathbb{C}$ is a (co)limit of a functor $J \rightarrow \mathbb{C}$. When $J$ are directed partial orders, respectively total orders, the $J$-colimits are called directed colimits, respectively inductive colimits.

A functor $L: J^{\prime} \rightarrow J$ is called final if for each object $j \in|J|$ the comma category $j / L$ is non-empty and connected. Consequently, a subcategory $J^{\prime} \subseteq J$ is final when the corresponding inclusion functor is final. Let us recall the following important result.

Theorem 1 [30] For each final functor $L: J^{\prime} \rightarrow J$ and each functor $D: J \rightarrow \mathbb{C}$, there exists a colimit $\mu: D \Rightarrow \operatorname{Colim}(D)$ and a canonical isomorphism $h: \operatorname{Colim}(L ; D) \rightarrow \operatorname{Colim}(D)$ when a colimit $\mu^{\prime}: L ; D \Rightarrow \operatorname{Colim}(L ; D)$ exists.

A class of arrows $\mathcal{S} \subseteq \mathbb{C}$ in a category $\mathbb{C}$ is stable under pushouts if for any pushout square in $\mathbb{C}$

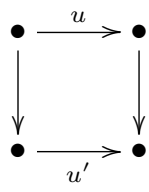

$u^{\prime} \in \mathcal{S}$ whenever $u \in \mathcal{S}$.

Given a class $\mathcal{D} \subseteq \mathbb{C}$ of arrows, which is closed under isomorphisms, an object $B$ is a $\mathcal{D}$-quotient representation of $A$ is there exists an arrow $(A \longrightarrow B) \in \mathcal{D}$. A D -quotient of $A$ is an isomorphism class of $\mathcal{D}$-quotient representations of $A$. $\mathbb{C}$ is $\mathcal{D}$-co-well-powered when for each object $A$, the $\mathcal{D}$ quotients of $A$ form a set.

A standard categorical approach to finiteness is provided by the concept of finitely presented object.

Definition 1 An object $A$ in a category $\mathbb{C}$ is finitely presented [1] if and only if the hom-functor $\mathbb{C}(A,-): \mathbb{C} \rightarrow$ Set preserves directed colimits.

For example a set is finitely presented (as object of Set) if and only if it is finite.

Definition 2 In any category $\mathbb{C}$, for any ordinal $\lambda$, a $\lambda$-chain is a commutative $\lambda$-diagram $\left(A_{i} \stackrel{f_{i, j}}{\rightarrow} A_{j}\right)_{i<j \leq \lambda}$ such that for each limit ordinal $\zeta \leq \lambda,\left(f_{i, \zeta}\right)_{i<\zeta}$ is the colimit of $\left(f_{i, j}\right)_{i<j<\zeta}$.

For any class of arrows $\mathcal{D} \subseteq \mathbb{C}$, a $(\lambda, \mathcal{D})$-chain is any $\lambda$-chain $\left(f_{i, j}\right)_{i<j \leq \lambda}$ such that $f_{i, i+1} \in \mathcal{D}$ for each $i<\lambda$.

We say that an arrow $h$ is a $(\lambda, \mathcal{D})$-chain if there exists a $(\lambda, \mathcal{D})$-chain $\left(f_{i, j}\right)_{i<j \leq \lambda}$ such that $h=f_{0, \lambda}$. In that case we may denote $f_{i, j}$ by $h_{i, j}$, for any $i<j \leq \lambda$.

\footnotetext{
1 Strictly speaking, this is only a hyper-category living in a higher set-theoretic universe.
} 


\subsection{Institutions}

An institution $\mathcal{I}=\left(\operatorname{Sig}^{\mathcal{I}}, \operatorname{Sen}^{\mathcal{I}}, \operatorname{Mod}^{\mathcal{I}}, \models^{\mathcal{I}}\right)$ consists of

1. a category $\mathbb{S} i g^{\mathcal{I}}$, whose objects are called signatures,

2. a functor $\operatorname{Sen}^{\mathcal{I}}: \mathbb{S i g}^{\mathcal{I}} \rightarrow \mathbb{S e t}$, giving for each signature a set whose elements are called sentences over that signature,

3. a functor $\operatorname{Mod}^{\mathcal{I}}:\left(\mathbb{S i g}^{\mathcal{I}}\right)^{\text {op }} \rightarrow \mathbb{C} \mathbb{A} \mathbb{T}$ giving for each signature $\Sigma$ a category whose objects are called $\Sigma$-models, and whose arrows are called $\Sigma$-(model) morphisms, and

4. a relation $\models{ }_{\Sigma}^{\mathcal{I}} \subseteq\left|\operatorname{Mod}^{\mathcal{I}}(\Sigma)\right| \times \operatorname{Sen}^{\mathcal{I}}(\Sigma)$ for each $\Sigma \in\left|\mathbb{S i g}^{\mathcal{I}}\right|$, called $\Sigma$-satisfaction,

such that for each morphism $\varphi: \Sigma \rightarrow \Sigma^{\prime}$ in $\mathbb{S i g}^{\mathcal{I}}$, the satisfaction condition

$$
M^{\prime} \models{ }_{\Sigma^{\prime}}^{\mathcal{I}} \operatorname{Sen}^{\mathcal{I}}(\varphi)(\rho) \text { iff } \operatorname{Mod}^{\mathcal{I}}(\varphi)\left(M^{\prime}\right) \models=_{\Sigma}^{\mathcal{I}} \rho
$$

holds for each $M^{\prime} \in\left|\operatorname{Mod}^{\mathcal{I}}\left(\Sigma^{\prime}\right)\right|$ and $\rho \in \operatorname{Sen}^{\mathcal{I}}(\Sigma)$. We denote the reduct functor $\operatorname{Mod}^{\mathcal{I}}(\varphi)$ by $-\uparrow_{\varphi}$ and the sentence translation $\operatorname{Sen}^{\mathcal{I}}(\varphi)$ by $\varphi\left({ }_{-}\right)$. When $M=M^{\prime} \uparrow_{\varphi}$ we say that $M$ is a $\varphi$-reduct of $M^{\prime}$, and that $M^{\prime}$ is a $\varphi$-expansion of $M$. When there is no danger of ambiguity, we may skip the superscripts from the notations of the entities of the institution; for example $\mathbb{S}_{i g} g^{\mathcal{I}}$ may be simply denoted $\mathbb{S} i g$.

General assumption: We assume that all our institutions are such that satisfaction is invariant under model isomorphism, i.e. if $\Sigma$-models $M, M^{\prime}$ are isomorphic, denoted $M \cong M^{\prime}$, then $M \models_{\Sigma} \rho$ iff $M^{\prime} \models_{\Sigma} \rho$ for all $\Sigma$-sentences $\rho$.

Example 1 Let FOL be the institution of first order logic with equality in its many sorted form.

Its signatures are triples $(S, F, P)$ consisting of

- a set of sort symbols $S$,

- a family $F=\left\{F_{w \rightarrow s} \mid w \in S^{*}, s \in S\right\}$ of sets of function symbols indexed by arities (for the arguments) and sorts (for the results), and

- a family $P=\left\{P_{w} \mid w \in S^{*}\right\}$ of sets of relation (predicate) symbols indexed by arities.

Signature morphisms map the three components in a compatible way. This means that a signature morphism $\varphi:(S, F, P) \rightarrow\left(S^{\prime}, F^{\prime}, P^{\prime}\right)$ consists of

- a function $\varphi^{\text {st }}: S \rightarrow S^{\prime}$,

- a family of functions $\varphi^{\mathrm{op}}=\left\{\varphi_{w \rightarrow s}^{\mathrm{op}}: F_{w \rightarrow s} \rightarrow F_{\varphi^{\text {st }}(w) \rightarrow \varphi^{\mathrm{st}}(s)}^{\prime} \mid w \in S^{*}, s \in S\right\}$, and

- a family of functions $\varphi^{\mathrm{rl}}=\left\{\varphi_{w \rightarrow s}^{\mathrm{rl}}: P_{w} \rightarrow P_{\varphi^{\mathrm{st}}(w)}^{\prime} \mid w \in S^{*}, s \in S\right\}$.

Models $M$ for a signature $(S, F, P)$ are first order structures interpreting each sort symbol $s$ as a set $M_{s}$, each function symbol $\sigma$ as a function $M_{\sigma}$ from the product of the interpretations of the argument sorts to the interpretation of the result sort, and each relation symbol $\pi$ as a subset $M_{\pi}$ of the product of the interpretations of the argument sorts. In order to avoid the existence of empty interpretations of the sorts, which may complicate unnecessarily our presentation, we assume that each signature has at least one constant (i.e. function symbol with empty arity) for each sort. A model homomorphism $h: M \rightarrow M^{\prime}$ is an indexed family of functions $\left\{h_{s}: M_{s} \rightarrow M_{s}^{\prime}\right\}_{s \in S}$ such that

- $h$ is an $F$-algebra homomorphism $M \rightarrow M^{\prime}$, i.e., $h_{s}\left(M_{\sigma}(m)\right)=M_{\sigma}^{\prime}\left(h_{w}(m)\right)$ for each $\sigma \in$ $F_{w \rightarrow s}$ and each $m \in M_{w}$, and

- $h_{w}(m) \in M_{\pi}^{\prime}$ if $m \in M_{\pi}$ (i.e. $h_{w}\left(M_{\pi}\right) \subseteq M_{\pi}^{\prime}$ ) for each relation $\pi \in P_{w}$ and each $m \in M_{w}$.

where $h_{w}: M_{w} \rightarrow M_{w}^{\prime}$ is the canonical component-wise extension of $h$, i.e. $h_{w}\left(m_{1}, \ldots, m_{n}\right)=$ $\left(h_{s_{1}}\left(m_{1}\right), \ldots, h_{s_{n}}\left(m_{n}\right)\right)$ for $w=s_{1} \ldots s_{n}$ and $m_{i} \in M_{s_{i}}$. A model homomorphism is $c l o s e d$ when $M_{\pi}=h_{w}^{-1}\left(M_{\pi}^{\prime}\right)$ for each relation symbol $\pi \in P_{w}$. 
For each signature morphism $\varphi$, the reduct $M^{\prime} \uparrow_{\varphi}$ of a model $M^{\prime}$ is defined by $\left(M^{\prime} \uparrow_{\varphi}\right)_{x}=M_{\varphi(x)}^{\prime}$ for each sort, function, or relation symbol $x$ from the domain signature of $\varphi$.

Sentences are the usual first order sentences built from equational and relational atoms by iterative application of Boolean connectives and quantifiers. Sentence translations along signature morphisms just rename the sorts, function, and relation symbols according to the respective signature morphisms. They can be formally defined by induction on the structure of the sentences. While the induction step is straightforward for the case of the Boolean connectives it needs a bit of attention for the case of the quantifiers. For any signature morphism $\varphi:(S, F, P) \rightarrow\left(S^{\prime}, F^{\prime}, P^{\prime}\right)$,

$$
\operatorname{Sen}^{\text {FOL }}(\varphi)((\forall X) \rho)=\left(\forall X^{\varphi}\right) \operatorname{Sen}^{\text {FOL }}\left(\varphi^{\prime}\right)(\rho)
$$

for each finite set $X$ of variables for $(S, F, P)$. The variables need to be disjoint from the constants of the signature, also we have to ensure that $\operatorname{Sen}^{\text {FOL }}$ thus defined is functorial indeed and that there is no overloading of variables (which in certain situations would cause a failure of the Satisfaction Condition). These may be formally achieved by considering that a variable for $(S, F, P)$ is a triple of the form $(x, s,(S, F, P))$ where $x$ is the name of the variable and $s \in S$ is the sort of the variable and that two different variables in $X$ have different names. Then we let $(S, F+X, P)$ be the extension of $(S, F, P)$ such that $(F+X)_{w \rightarrow s}=F_{w \rightarrow s}$ when $w$ is non-empty and $(F+X)_{\rightarrow s}=$ $F_{\rightarrow s} \cup\{(x, s,(S, F, P)) \mid(x, s,(S, F, P)) \in X\}$ and we let $\varphi^{\prime}:(S, F+X, P) \rightarrow\left(S^{\prime}, F^{\prime}+X^{\varphi}, P^{\prime}\right)$ be the canonical extension of $\varphi$ that maps each variable $(x, s,(S, F, P))$ to $\left(x, \varphi(s),\left(S^{\prime}, F^{\prime}, P^{\prime}\right)\right)$.

The satisfaction of sentences by models is the usual Tarskian satisfaction defined inductively on the structure of the sentences.

An universal Horn sentence in FOL for a first order signature $(S, F, P)$ is a sentence of the form $(\forall X)(H \Rightarrow C)$, where $H$ is a finite conjunction of (relational or equational) atoms and $C$ is a (relational of equational) atom, and $H \Rightarrow C$ is the implication of $C$ by $H$. The 'sub-institution' HCL, Horn clause logic, of FOL has the same signature category and model functor as FOL but only universal Horn sentences as sentences.

An algebraic signature $(S, F)$ is just a FOL signature without relation symbols. The 'subinstitution' of HCL which restricts the signatures only to the algebraic ones and the sentences to universally quantified equations is called equational logic and is denoted by EQL.

Example 2 Here we consider the institution PA of partial algebra as employed by the specification language CASL [3].

A partial algebraic signature is a tuple $(S, T F, P F)$, where $T F$ is a family of sets of total function symbols and $P F$ is a family of sets of partial function symbols such that $T F_{w \rightarrow s} \cap P F_{w \rightarrow s}=\emptyset$ for each arity $w$ and each sort $s$. In order to avoid empty carriers, like in the case of FOL, we assume there exists at least one total constant for each sort. Signature morphisms map the three components in a compatible way.

A partial algebra is just like an ordinary algebra (i.e. a FOL model without relations) but interpreting the function symbols of $P F$ as partial rather than total functions. A partial algebra homomorphism $h: A \rightarrow B$ is a family of (total) functions $\left\{h_{s}: A_{s} \rightarrow B_{s}\right\}_{s \in S}$ indexed by the set of sorts $S$ of the signature such that $h_{s}\left(A_{\sigma}(a)\right)=B_{\sigma}\left(h_{w}(a)\right)$ for each function symbol $\sigma \in T F_{w \rightarrow s} \cup P F_{w \rightarrow s}$ and each string of arguments $a \in A_{w}$ for which $A_{\sigma}(a)$ is defined.

The sentences have three kinds of atoms: definedness $\operatorname{def}(t)$, strong equality $t \stackrel{s}{=} t^{\prime}$, and existence equality $t \stackrel{e}{=} t^{\prime}$. The definedness $\operatorname{def}(t)$ of a term $t$ holds in a partial algebra $A$ when the interpretation $A_{t}$ of $t$ is defined. The strong equality $t \stackrel{s}{=} t^{\prime}$ holds when both terms are undefined or both of them are defined and are equal. The existence equality $t \stackrel{e}{=} t^{\prime}$ holds when both terms are defined and are equal. ${ }^{2}$ The sentences are formed from these atoms by Boolean connectives and quantifications over total variables (i.e variables that are always defined).

\footnotetext{
${ }^{2}$ Notice that $\operatorname{def}(t)$ is equivalent to $t \stackrel{e}{=} t$ and that $t \stackrel{s}{=} t^{\prime}$ is equivalent to $\left(t \stackrel{e}{=} t^{\prime}\right) \vee\left(\neg \operatorname{def}(t) \wedge \neg \operatorname{def}\left(t^{\prime}\right)\right)$.
} 
Example 3 Preordered algebras are used for formal specification and verifications of algorithms [18], for automatic generation of case analysis [18], and in general about reasoning about transitions between states of systems. They constitute an unlabeled form of rewriting logic of [32]. Let POA denote the institution of preordered algebras.

The signatures are just ordinary algebraic signatures. The POA models are preordered algebras which are interpretations of the signatures into the category of preorders $\mathbb{P}$ re rather than the category of sets Set. This means that each sort gets interpreted as a preorder, and each function symbol as a monotonic function. A preordered algebra homomorphism is just a family of monotonic functions which is an algebra homomorphism.

The sentences have two kinds of atoms: (ordinary) equations and preorder atoms. A preorder atom $t \leq t^{\prime}$ is satisfied by a preordered model $M$ when the interpretations of the terms are in the preorder relation of the carrier, i.e. $M_{t} \leq M_{t^{\prime}}$. The sentences are formed from these atoms by Boolean connectives and quantifications over variables.

Other examples of institutions in use in computing science include higher-order [5], polymorphic [39], various modal logics such as temporal [20], process [20], behavioral [4], coalgebraic [7], object-oriented [22], and multi-algebraic (non-determinism) [29] logics.

For any signature $\Sigma$ in an institution $\mathcal{I}$ :

- For each set $E$ of $\Sigma$-sentences, let $E^{*}=\left\{M \in \operatorname{Mod}(\Sigma)|M|_{\Sigma} e\right.$ for each $\left.e \in E\right\}$, and

- For each class $\mathbb{M}$ of $\Sigma$-models, let $\mathbb{M}^{*}=\left\{e \in \operatorname{Sen}(\Sigma) \mid M \models_{\Sigma} e\right.$ for each $\left.M \in \mathbb{M}\right\}$.

For an individual sentence or model $x$, by $x^{*}$ we mean $\{x\}^{*}$.

If $E$ and $E^{\prime}$ are sets of sentences of the same signature, then $E^{\prime} \subseteq E^{* *}$ is denoted by $E \models E^{\prime}$.

Two sentences, $\rho_{1}$ and $\rho_{2}$ of the same signature are semantically equivalent, denoted $\models \mid$ when $\rho_{1}=\rho_{2}$ and $\rho_{2} \models \rho_{1}$. Two models, $M_{1}$ and $M_{2}$ of the same signature are elementarily equivalent, denoted $M_{1} \equiv M_{2}$, when they satisfy the same sentences, i.e. $\left\{M_{1}\right\}^{*}=\left\{M_{2}\right\}^{*}$.

Definition 3 [16] In any institution a $\Sigma$-sentence $\rho$ is finitary if and only if it can be written as $\varphi\left(\rho_{0}\right)$ where $\varphi: \Sigma_{0} \rightarrow \Sigma$ is a signature morphism such that $\Sigma_{0}$ is a finitely presented signature and $\rho_{0}$ is a $\Sigma_{0}$-sentence.

An institution has finitary sentences when all its sentences are finitary.

The above concept is a categorical expression of the fact that sentences contain only a finite number of symbols. FOL, PA, POA are all examples of institutions with finitary sentences. For example in FOL, a signature $(S, F, P)$ is finitely presented if and only if $S, F$, and $P$ are finite. (Here $F$ 'finite' means that $\left\{(w, s) \mid F_{w \rightarrow s} \neq \emptyset\right\}$ is finite and each $F_{w \rightarrow s}$ is also finite, and analogously for $P$.) Consequently, it is obvious that FOL has finitary sentences.

\subsection{Model amalgamation, Elementary diagrams, Internal logic}

The rest of this preliminary section is devoted to a brief overview of some of the fundamental concepts and methods in institution-independent model theory.

\subsubsection{Model amalgamation}

Exactness properties for institutions formalize the possibility of amalgamating models of different signatures when they are consistent on some kind of 'intersection' of the signatures (formalized as a pushout square). An institution $\mathcal{I}$ is exact if and only if the model functor $\operatorname{Mod}^{\mathcal{I}}:\left(\mathbb{S i g}^{\mathcal{I}}\right)^{\text {op }} \rightarrow \mathbb{C} \mathbb{T}$ preserves finite limits. The institution is semi-exact if and only if $\operatorname{Mod}^{\mathcal{I}}$ preserves pullbacks.

Semi-exactness is everywhere. Virtually all institutions formalizing conventional or non-conventional logics are at least semi-exact. In general the institutions of many-sorted logics are exact, while those 
of unsorted (or one-sorted) logics are only semi-exact [19]. However, in applications the important amalgamation property is the semi-exactness rather than the full exactness. Moreover, in practice often the weak ${ }^{3}$ version of exactness suffices [10,44,34].

The following amalgamation property is a direct consequence of semi-exactness. The commuting square of signature morphisms

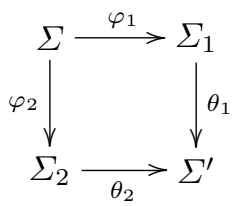

is an amalgamation square if and only if for each $\Sigma_{1}$-model $M_{1}$ and a $\Sigma_{2}$-model $M_{2}$ such that $M_{1} \uparrow_{\varphi_{1}}=M_{2} \uparrow_{\varphi_{2}}$, there exists an unique $\Sigma^{\prime}$-model $M^{\prime}$, denoted $M_{1} \otimes_{\varphi_{1}, \varphi_{2}} M_{2}$, such that $M^{\prime} \uparrow_{\theta_{1}}=$ $M_{1}$ and $M^{\prime} \uparrow_{\theta_{2}}=M_{2}$. When there is no danger of confusion we denote $M_{1} \otimes_{\varphi_{1}, \varphi_{2}} M_{2}$ by the simpler notation $M_{1} \otimes M_{2}$. We can notice easily that in a semi-exact institution each pushout square of signature morphisms is an amalgamation square.

More generally, for $J$ small category, an institution is $J$-exact when $\operatorname{Mod}^{\mathcal{I}}$ maps $J$-colimits of signatures to corresponding limits of categories of models. In particular, an institution is directed/inductiveexact when Mod maps directed/inductive colimits of signatures to corresponding limits of categories of models.

\subsubsection{The method of diagrams}

The method of diagrams is one of the most important conventional model theoretic methods. At the level of institution-independent model theory, cf. [12] this is reflected as a categorical property which formalizes the idea that the class of model morphisms from a model $M$ can be represented (by a natural isomorphism) as a class of models of a theory in a signature extending the original signature with syntactic entities determined by $M$. Elementary diagrams can be seen as a coherence property between the semantic structure and the syntactic structure of an institution. By following the basic principle that a structure is rather defined by its homomorphisms (arrows) than by its objects, the semantical structure of an institution is given by its model homomorphisms. On the other hand the syntactical structure of an institution is essentially determined by its atomic sentences.

According to [12], an institution $\mathcal{I}$ has elementary diagrams when for each signature $\Sigma$ and each $\Sigma$-model $M$, there exists a signature $\Sigma_{M}$ and a signature morphism $\iota_{\Sigma}(M): \Sigma \rightarrow \Sigma_{M}$, functorial in $\Sigma$ and $M$, and a set $E_{M}$ of $\Sigma_{M}$-sentences such that $\operatorname{Mod}\left(\Sigma_{M}, E_{M}\right)$ and the comma category $M / \operatorname{Mod}(\Sigma)$ are naturally isomorphic, i.e. the following diagram commutes by the isomorphism $i_{\Sigma, M}$ that is natural in $\Sigma$ and $M$.

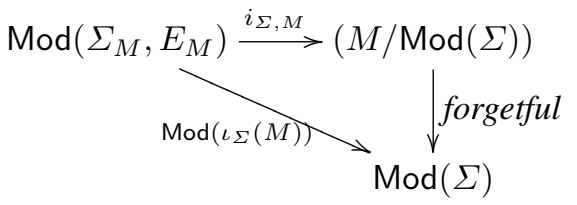

The "functoriality" of $\iota$ means that for each signature morphism $\varphi: \Sigma \rightarrow \Sigma^{\prime}$ and each $\Sigma$-model homomorphism $h: M \rightarrow M^{\prime} \uparrow_{\varphi}$, there exists a signature morphism $\iota_{\varphi}(h): \Sigma_{M} \rightarrow \Sigma_{M^{\prime}}^{\prime}$ such that $E_{M^{\prime}} \models \iota_{\varphi}(h)\left(E_{M}\right)$ and such that

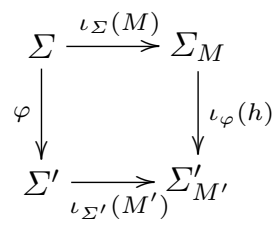

\footnotetext{
3 In the sense of 'weak' universal properties [30] not requiring uniqueness.
} 
commutes and $\iota_{\varphi}(h) ; \iota_{\varphi^{\prime}}\left(h^{\prime}\right)=\iota_{\varphi ; \varphi^{\prime}}\left(h ; h^{\prime} \uparrow_{\varphi}\right)$ and $\iota_{1_{\Sigma}}\left(1_{M}\right)=1_{\Sigma_{M}}$.

The "naturality" of $i$ means that for each signature morphism $\varphi: \Sigma \rightarrow \Sigma^{\prime}$ and each $\Sigma$-model homomorphism $h: M \rightarrow M^{\prime} \uparrow_{\varphi}$ the following diagram commutes:

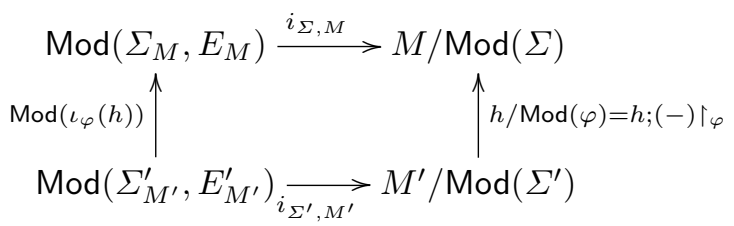

The signature morphism $\iota_{\Sigma}(M): \Sigma \rightarrow \Sigma_{M}$ is called the elementary extension of $\Sigma$ via $M$ and the set $E_{M}$ of $\Sigma_{M}$-sentences is called the elementary diagram of the model $M$. Note that $i_{\Sigma, M}^{-1}\left(1_{M}\right)$ is the initial model of $\left(\Sigma_{M}, E_{M}\right)$, which we denote as $M_{M}$.

Example 4 For any $(S, F, P)$-model $M$, let $\left(F_{M}\right)_{\rightarrow s}=F_{\rightarrow s} \cup M_{s}$, otherwise let $\left(F_{M}\right)_{w \rightarrow s}=$ $F_{w \rightarrow s}$, and let $M_{M}$ be the $\left(S, F_{M}, P\right)$-expansion of $M$ such that $M_{m}=m$ for each $m \in M$. Then $E_{M}$ is the set of all (relational or equational) atoms satisfied by $M_{M}$.

However, depending on the chosen concept of model homomorphism one may get other elementary diagrams for FOL. For example, when one restricts model homomorphisms to injective ones, $E_{M}$ consists of all atoms and negations of atomic equations satisfied by $M_{M}$, when one restricts them to the closed ones if $M_{\pi}=h^{-1}\left(N_{\pi}\right)$ for each $\left.\pi \in P\right), E_{M}$ consists of all atoms and negations of atomic relations satisfied by $M_{M}$, and when one restricts them to closed injective model homomorphisms, $E_{M}$ consists of all atoms and all negations of atoms satisfied by $M_{M}$. Let us recall that an $(S, F, P)$-homomorphism $h: M \rightarrow N$ is an elementary embedding when $N \models \rho$ implies $M \models \rho$ for each $(S, F, P)$-sentence $\rho$. When model homomorphisms are restricted to elementary embeddings, then $E_{M}=M_{M}^{*}$.

Example 5 The institution PA of partial algebras has elementary diagrams such that given a partial algebra $A$, the elementary extension $\iota(A)$ of its signature via $A$ adds its elements as total constants and the elementary diagram $E_{A}$ of $A$ consists of all existence equations satisfied by $A_{A}$, where $A_{A}$ is the $\iota(A)$-expansion of $A$ interpreting each of its elements by itself.

Example 6 POA has elementary diagrams such that given a preorder algebra $M$, the elementary extension $\iota(M)$ of its signature via $M$ adds its elements as constants and the elementary diagram $E_{M}$ of $M$ consists of all equations and preorder atoms satisfied by $M_{M}$, where $M_{M}$ is the $\iota(M)$ expansion of $M$ interpreting each of its elements by itself.

In similar ways, many other institutions either from conventional logic or from computing science, have elementary diagrams $[12,17]$. The institution-independent concept of elementary diagrams presented above has been successfully used in a rather crucial way for developing several results in institution-independent model theory, including (quasi-)variety theorems and existence of free models for theories [12,17], Robinson consistency and Craig interpolation [26], Tarski elementary chain theorem [25], existence of (co)limits of theory models [12], etc. A quite different predecessor of our institution-independent method of diagrams has been used for developing quasivariety theorems and existence of free models within the context of the so-called 'abstract algebraic institutions' $[42,43]$.

\subsubsection{Internal logic}

Much of our institution-independent development of model theory relies on the possibility of defining concepts such as classical Boolean connectives, quantification, and atomic sentences internally to any institution. The main implication of this fact is that the abstract satisfaction relation between 
models and sentences can be decomposed at the level of arbitrary institutions into several concrete layers of satisfaction defined categorically in terms of (a simple form of) injectivity and reduction. Essentially speaking, this is what gives depth to the institution-independent approach to model theory.

Definition $4[41,11]$ Given a signature $\Sigma$ in an institution

- the $\Sigma$-sentence $\rho^{\prime}$ is a (semantic) negation of $\rho$ when $\rho^{\prime *}=|\operatorname{Mod}(\Sigma)| \backslash \rho^{*}$, and

- the $\Sigma$-sentence $\rho^{\prime}$ is the (semantic) conjunction of the $\Sigma$-sentences $\rho_{1}$ and $\rho_{2}$ when $\rho^{\prime *}=\rho_{1}^{*} \cap \rho_{2}^{*}$.

An institution has (semantic) negation when each sentence of the institution has a negation, and has (semantic) conjunctions when each two sentences (of the same signature) have a conjunction. Distinguished negations are often denoted by $\neg_{-}$, while distinguished conjunctions by $\_\wedge_{-}$.

Other Boolean connectives, such as disjunction $(\vee)$, implication $(\Rightarrow)$, equivalence $(\Leftrightarrow)$, etc., can be derived as it is usually classically done from negations and (finite) conjunctions.

An institution which has negations and finite conjunctions is called a Boolean complete institution.

Fact 21 The Boolean connectives are unique modulo semantical equivalence.

Definition $5[43,11]$ For any signature morphism $\chi: \Sigma \rightarrow \Sigma^{\prime}$ in an arbitrary institution,

- a $\Sigma$-sentence $\rho$ is a (semantic) existential $\chi$-quantification of a $\Sigma^{\prime}$-sentence $\rho^{\prime}$ when $\rho^{*}=$ $\left(\rho^{\prime *}\right) \uparrow_{\chi}$; in this case we may write $\rho$ as $(\exists \chi) \rho^{\prime}$,

- a $\Sigma$-sentence $\rho$ is a (semantic) universal $\chi$-quantification of a $\Sigma^{\prime}$-sentence $\rho^{\prime}$ when $\rho^{*}=$ $\mid \operatorname{Mod}(\Sigma) \backslash\left(\left|\operatorname{Mod}\left(\Sigma^{\prime}\right)\right| \backslash \rho^{\prime *}\right) \uparrow_{\chi}$; in this case we may write $\rho$ as $(\forall \chi) \rho^{\prime}$.

For a class $\mathcal{D} \subseteq \mathbb{S i g}$ of signature morphisms, we say that the institution has universal/existential $\mathcal{D}$-quantification when for each $\chi: \Sigma \rightarrow \Sigma^{\prime}$ in $\mathcal{D}$, each $\Sigma^{\prime}$-sentence has a universal/existential $\chi$-quantification.

Example 7 FOL has $\mathcal{D}$-quantifications for the class $\mathcal{D}$ of the injective signature extensions with a finite number of constants, which means the class of the signature morphisms $\chi: \Sigma \rightarrow \Sigma^{\prime}$ that are injective in all components and such that $\Sigma^{\prime}$ has only constants outside the image of $\varphi$.

Let $\chi: \Sigma \rightarrow \Sigma^{\prime}$ be a signature morphism as above and let $\rho^{\prime}$ be a $\Sigma^{\prime}$-sentence. Then $(\forall \chi) \rho^{\prime}$ is the $\Sigma$ sentence defined as follows. There exists a signature extension $\chi^{\prime}: \Sigma \rightarrow \Sigma^{\prime \prime}$ of $\Sigma$ with a finite set of variables $X$ such that there exists an isomorphism of signatures (i.e. bijective component-wise) $\theta: \Sigma^{\prime} \rightarrow \Sigma^{\prime \prime}$ with $\chi ; \theta=\chi^{\prime}$. We define $(\forall \chi) \rho^{\prime}$ as the FOL-sentence $(\forall X) \theta\left(\rho^{\prime}\right)$. The same kind of argument applies also to existential quantifications in FOL.

PA has $\mathcal{D}$-quantification for $\mathcal{D}$ the class of the injective signature extensions with a finite number of total constants, while POA has quantification similar to FOL.

Second order quantification can be captured by taking $\mathcal{D}$ to be the class of the injective signature morphisms.

Note that all the classes $\mathcal{D}$ of signature morphisms introduced in this example enjoy properties such as closure under composition, under isomorphisms, and stability under pushouts.

\subsubsection{Finitary, small, quasi-representable signature morphisms}

In many actual institutions, quantifications are done via signature extensions with a finite number of constants. The following definition generalizes this situation to signature morphisms in arbitrary institutions, and further to infinite cardinalities. 
Definition 6 A signature morphism $\chi: \Sigma \rightarrow \Sigma^{\prime}$ is finitary when for each co-limit $\left(\mu_{i}\right)_{i \in I}$ of a directed diagram $\left(f_{i, j}\right)_{(i<j) \in(I, \leq)}$ of $\Sigma$-models

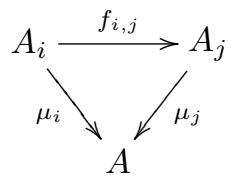

and for each $\chi$-expansion $A^{\prime}$ of $A$

- there exists an index $i \in I$ and a $\chi$-expansion $\mu_{i}^{\prime}: A_{i}^{\prime} \rightarrow A^{\prime}$ of $\mu_{i}$, and

- any two different expansions as above can be 'unified' in the sense that for any $\chi$-expansions $\mu_{i}^{\prime}$ and $\mu_{k}^{\prime}$ as above there exists an index $j \in I$ with $i, k<j$, a $\chi$-expansion $\mu_{j}^{\prime}$ as above and $f_{i, j}^{\prime}, f_{k, j}^{\prime} \chi$-expansions of $f_{i, j}, f_{k, j}$ such that the following commutes

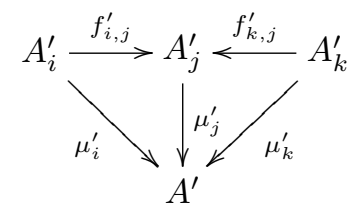

A signature morphism $\Sigma \stackrel{\varphi}{\longrightarrow} \Sigma^{\prime}$ is $\lambda$-small for a cardinal $\lambda$ when for each $\lambda$-chain $\left(M_{i} \stackrel{f_{i, j}}{\longrightarrow} M_{j}\right)_{i<j \leq \lambda}$

of $\Sigma$-homomorphisms and each $M^{\prime}$ a $\varphi$-expansion of $M_{\lambda}$, there exists $i<\lambda$ and $M_{i}^{\prime} \stackrel{f_{i, \lambda}^{\prime}}{\longrightarrow} M^{\prime}$ a $\varphi$-expansion of $f_{i, \lambda}$.

Fact 22 Finitary signature morphisms are $\lambda$-small for each infinite cardinal $\lambda$.

The concept of quasi-representable signature morphisms defined below are a slight weakening of the concept of representable signature morphisms introduced in [11] which capture the signature extensions with constants in an abstract categorical manner.

Definition $7[17,25]$ In any institution, a signature morphism $\chi: \Sigma \rightarrow \Sigma^{\prime}$ is quasi-representable when for each $\Sigma^{\prime}$-model $M^{\prime}$, the canonical functor below determined by the reduct functor $\operatorname{Mod}(\chi)$ is an isomorphism (of comma categories)

$$
M^{\prime} / \operatorname{Mod}\left(\Sigma^{\prime}\right) \cong\left(M^{\prime} \uparrow_{\chi}\right) / \operatorname{Mod}(\Sigma) \text {. }
$$

Example 8 Usual first order variables in actual standard institutions such as FOL, PA, POA, but also in institutions such as $E($ FOL), the restriction of FOL allowing only elementary embeddings as model homomorphisms, as captured by the classes $\mathcal{D}$ of signature morphisms of Ex. 5, provide examples of quasi-representable signature morphisms. However, this concept accommodates also other less conventional types of variables (see [17]).

The proof of Propositions 1 and 2 below are given in the appendix.

Proposition 1 All model reduct functors corresponding to quasi-representable signature morphisms create directed colimits of models.

The following shows some important structural properties of quasi-representability.

\section{Proposition 2}

1. In any institution the (finitary) quasi-representable signature morphisms form a subcategory of Sig.

2. If the institution is semi-exact, then quasi-representable signature morphisms are stable under pushouts.

3. If the institution is directed-exact, then any directed colimit of quasi-representable signature morphisms consists of quasi-representable signature morphisms.

4. If $\varphi$ and $\varphi ; \chi$ are quasi-representable then $\chi$ is quasi-representable. 


\section{Saturated Models: existence}

Definition 8 For each signature morphism $\chi: \Sigma \rightarrow \Sigma^{\prime}$, a $\Sigma$-model $M \chi$-realizes a set $E^{\prime}$ of $\Sigma^{\prime}$ sentences, if there exists a $\chi$-expansion $M^{\prime}$ of $M$ which satisfies $E^{\prime}$. It $\chi$-realizes finitely $E^{\prime}$ if it realizes every finite subset of $E^{\prime}$.

A $\Sigma$-model $M$ is $(\lambda, \mathcal{D})$-saturated for $\lambda$ a cardinal and $\mathcal{D}$ a class of signature morphisms when for each ordinal $\alpha<\lambda$ and each $(\alpha, \mathcal{D})$-chain $\left(\Sigma_{i} \stackrel{\varphi_{i, j}}{>} \Sigma_{j}\right)_{i<j \leq \alpha}$ with $\Sigma_{0}=\Sigma$, for each $\left(\Sigma_{\alpha} \stackrel{\chi}{\longrightarrow} \Sigma^{\prime}\right) \in \mathcal{D}$, each $\varphi_{0, \alpha}$-expansion of $M \chi$-realizes any set of sentences if and only if it $\chi$-realizes it finitely.

Example 9 The conventional concept of saturated model (see Sect. 5.1 of [6]) is an instance of Dfn. 8 by taking $\mathcal{D}$ to be the class of FOL injective signature extensions with one constant. Note than one reaches the same when replacing 'one constant' by 'finite number of constants'. The latter variant for $\mathcal{D}$ has better structural properties, such as closure under composition.

Fact 31 Let $\mathcal{D}$ be the class of FOL injective signature extensions with a finite number of constants, and let $\lambda$ be an infinite cardinal. For each $(\lambda, \mathcal{D})$-saturated model $M$ and for each sort $s$, if $M_{s}$ is infinite then $\operatorname{card}\left(M_{s}\right) \geq \lambda$.

Proof Let $\Sigma$ be the (FOL) signature of $M$. Let us assume that $\operatorname{card}\left(M_{s}\right)<\lambda$ for some sort $s$ for which $M_{s}$ is infinite. Then we take one $\left(\operatorname{card}\left(M_{s}\right), \mathcal{D}\right)$-chain given by the signature extension with constants $\Sigma \hookrightarrow \Sigma \uplus M_{s}$, and let $\chi$ be the extension of $\Sigma \uplus M_{s}$ with one new constant $x$. Consider $E=\left\{x \neq m \mid m \in M_{s}\right\}$. Then the $\left(\Sigma \uplus M_{s}\right)$-expansion $M^{\prime}$ of $M$ such that $M_{m}^{\prime}=m$ for each $m \in M_{s}$, finitely realizes $E$ but does not realize $E$, which contradicts the fact that $M$ is $(\lambda, \mathcal{D})$-saturated.

The following result shows that each model can be elementarily 'extended' into a saturated model, thus giving the existence of saturated models. This existence result comes up with a set of merely technical conditions, which can be rather easily established in the concrete examples, and that are sufficient to lift the only fundamental assumption, namely the 5th condition of Thm. 2, through $(\lambda, \mathcal{D})$-chains. This core condition can be regarded as a form of compactness.

Theorem 2 Consider an institution and a class $\mathcal{D}$ of signature morphisms that is closed under compositions with isomorphisms and such that

1. $M \equiv N$ if there exists a model homomorphism $M \rightarrow N$,

2. it has finite conjunctions and existential D-quantifications,

3. it has inductive colimits of signatures and is inductive-exact,

4. for each signature $\Sigma$, the category of $\Sigma$-models has inductive colimits,

5. for each signature morphism $\Sigma \stackrel{\chi}{\longrightarrow} \Sigma^{\prime} \in \mathcal{D}$ and $E^{\prime}$ set of $\Sigma^{\prime}$-sentences, if $A$ realizes $E^{\prime}$ finitely then there exists a model homomorphism $A \rightarrow B$ such that $B$ realizes $E^{\prime}$,

6. for each signature morphism $\Sigma \stackrel{\chi}{\longrightarrow} \Sigma^{\prime} \in \mathcal{D}$ and each $\Sigma$-model $M$, the class of $\chi$-expansions of $M$ form a set, and

7. each signature morphism from $\mathcal{D}$ is quasi-representable, the category $\mathbb{S} i g$ of signatures is $\mathcal{D}$-cowell-powered, and for each ordinal $\lambda$ there exists a cardinal $\alpha$ such that each morphism that is $a(\lambda, \mathcal{D})$-chain is $\alpha$-small.

Then for any cardinal $\lambda$ and for each $\Sigma$-model $M$ there exists a $\Sigma$-homomorphism $M \rightarrow N$ such that $N$ is $(\lambda, \mathcal{D})$-saturated.

Proof First we prove that there exists a $\Sigma$-homomorphism $M \stackrel{h}{\longrightarrow} N$ such that for each $(\lambda, \mathcal{D})$ chain $\Sigma \stackrel{\varphi}{\longrightarrow} \Sigma^{\prime}$, each $\left(\Sigma^{\prime} \stackrel{\chi}{\longrightarrow} \Sigma^{\prime \prime}\right) \in \mathcal{D}$, each $\varphi$-expansion $M^{\prime}$ of $M$, and each set $E^{\prime \prime}$ of $\Sigma^{\prime \prime}$ sentences realized finitely by $M^{\prime}, N^{\prime} \chi$-realizes $E^{\prime \prime}$, where $M^{\prime} \stackrel{h^{\prime}}{\longrightarrow} N^{\prime}$ is the unique $\varphi$-expansion 
of $h$. (The existence of $h^{\prime}$ is guaranteed by the fact that $\varphi$ is quasi-representable, which follows by ordinal induction from the condition that all signature morphisms in $\mathcal{D}$ are quasi-representable and that the institution is inductive-exact, by applying Prop. 2 [for inductive colimits rather than the more general directed colimits].)

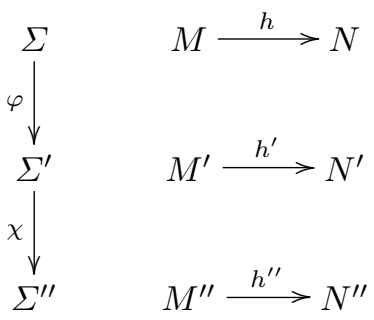

For fixed $\Sigma$ and $M$, by $\left(\varphi, M^{\prime}, \chi, E^{\prime \prime}\right)$ let us denote tuples where $\Sigma \stackrel{\varphi}{\longrightarrow} \Sigma^{\prime}$ is a $(\lambda, \mathcal{D})$-chain, $M^{\prime}$ is a $\varphi$-expansion of $M,\left(\Sigma^{\prime} \stackrel{\chi}{\longrightarrow} \Sigma^{\prime \prime}\right) \in \mathcal{D}$, and $E^{\prime \prime}$ is a set of $\Sigma^{\prime \prime}$-sentences which is $\chi$ realized finitely by $M^{\prime}$. Two such tuples $\left(\varphi^{1}, M^{\prime}, \chi^{1}, E^{1}\right)$ and $\left(\varphi^{2}, M^{\prime 2}, \chi^{2}, E^{2}\right)$ are isomorphic when there exists an isomorphism $\theta: \varphi^{1} ; \chi^{1} \Rightarrow \varphi^{2} ; \chi^{2}$ of $(\lambda+1)$-chains

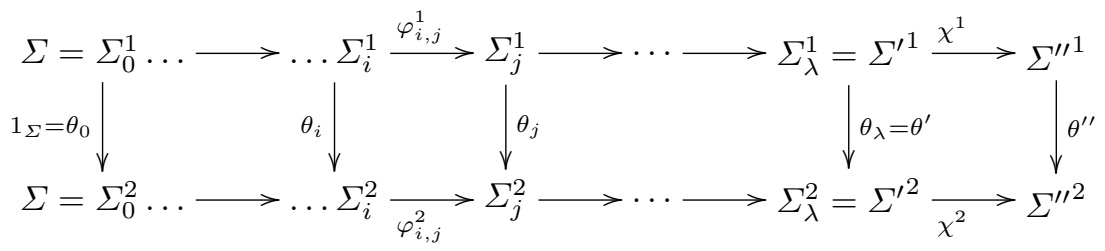

such that $M^{\prime 2} \uparrow_{\theta^{\prime}}=M^{\prime 1}$ and $\theta^{\prime \prime}\left(E^{1}\right)=E^{2}$. By the conditions of the theorem ( $\operatorname{Sig}$ being $\mathcal{D}$-cowell-powered and all $\chi$-expansions of a model forming a set), the isomorphism classes of tuples $\left(\varphi, M^{\prime}, \chi, E^{\prime \prime}\right)$ form a set, let us denote it by $L(M)$. If $k$ is the cardinal of $L(M)$, we may consider $\left\{\left(\varphi^{i}, M^{\prime}, \chi^{i}, E^{i}\right) \mid i<k\right\}$ a complete system of independent representatives for $L(M)$.

Now, by ordinal induction we construct a chain of $\Sigma$-homomorphisms $\left(M_{i} \stackrel{h_{i, j}}{\longrightarrow} M_{j}\right)_{i<j \leq k}$ as follows:

- $M_{0}=M$

- for each successor ordinal $j+1$ let $M^{\prime j} \stackrel{h_{0, j}^{\prime}}{\longrightarrow} M_{j}^{\prime}$ be the unique $\varphi^{j}$-expansion of $M \stackrel{h_{0, j}}{\longrightarrow} M_{j}$. Because $M^{\prime j} \chi^{j}$-realizes $E^{j}$ finitely, we have that $M_{j}^{\prime} \chi^{j}$-realizes $E^{j}$ finitely too. By condition

5., there exists $M_{j}^{\prime} \stackrel{f^{\prime}}{\longrightarrow} P^{\prime}$ such that $P^{\prime} \chi^{j}$-realizes $E^{j}$. Then we define $M_{j+1}=P^{\prime} \uparrow_{\varphi^{j}}$ and $h_{j, j+1}=f^{\prime} \uparrow_{\varphi^{j}}$, and

- for each limit ordinal $j$ we take the colimit of the chain before $j$.

Let $N=M_{k}$ and $h=h_{0, k}$. Keeping above notations, consider $\left(\varphi, M^{\prime}, \chi, E^{\prime \prime}\right)$. If $j<k$ is the isomorphism class of $\left(\varphi, M^{\prime}, \chi, E^{\prime \prime}\right)$, we may assume without any loss of generality that $\left(\varphi, M^{\prime}, \chi, E^{\prime \prime}\right)=$ $\left(\varphi^{j}, M^{\prime j}, \chi^{j}, E^{j}\right)$. We have to show that $N^{\prime} \chi^{j}$-realizes $E^{j}$.

This holds because we have that $M_{j+1}^{\prime} \chi^{j}$-realizes $E^{j}$ (where $M_{j+1}^{\prime}$ is the unique $\varphi^{j}$-expansion of $M_{j+1}$ determined by $M^{\prime j} \uparrow_{\varphi_{j}}=M \stackrel{h_{0, j+1}}{\rightarrow} M_{j+1}$.). Let $M_{j+1}^{\prime \prime}$ be a $\chi^{j}$-expansion of $M_{j+1}^{\prime}$ such that $M_{j+1}^{\prime \prime} \models E^{j}$. Because $M_{j+1}^{\prime \prime} \stackrel{h_{j+1, k}^{\prime \prime}}{\longrightarrow} N^{\prime \prime}$, the unique $\left(\varphi^{j} ; \chi^{j}\right)$-expansion of $h_{j+1, k}$, preserves satisfaction $N^{\prime \prime} \models E^{j}$, hence $N^{\prime} \chi^{j}$-realizes $E^{j}$.

In the second part of the proof we assume the conclusion of the first part and consider a cardinal $\alpha$ such that each $(\lambda, \mathcal{D})$-chain is $\alpha$-small. By ordinal induction we construct a $\alpha$-chain $\left(N_{i} \stackrel{f_{i, j}}{\longrightarrow} N_{j}\right)_{i<j \leq \alpha}$ 
such that $N_{0}=M$ and each $f_{j, j+1}$ has the property of $h$ above. We want to show that $N_{\alpha}$ is $(\lambda, \mathcal{D})$ saturated, therefore the desired model homomorphism is $M \stackrel{f_{0, \alpha}}{\longrightarrow} N_{\alpha}$.

Assume $N_{\alpha}^{\prime} \chi$-realizes $E^{\prime \prime}$ finitely, where $\left(\varphi, N_{\alpha}^{\prime}, \chi, E^{\prime \prime}\right) \in L\left(N_{\alpha}\right)$. We have to prove that $N_{\alpha}^{\prime}$ $\chi$-realizes $E^{\prime \prime}$. Because $\varphi$ is $\alpha$-small, there exists $j<\alpha$ and $N_{j}^{\prime} \stackrel{f_{j, \alpha}^{\prime}}{\longrightarrow} N_{\alpha}^{\prime}$ a $\varphi$-expansion of $f_{j, \alpha}$. By quasi-representability this determines expansions $N_{j}^{\prime} \stackrel{f_{j, j+1}^{\prime}}{\longrightarrow} N_{j+1}^{\prime}$ and $N_{j+1}^{\prime} \stackrel{f_{j+1, \alpha}^{\prime}}{\longrightarrow} N_{\alpha}^{\prime}$. Notice that by conditions 1 . and 2., $N_{j}^{\prime} \chi$-realizes finitely $E^{\prime \prime}$ because $N_{\alpha}^{\prime}$ does. Recall that $f_{j, j+1}$ has the property of $h$ from the first part of the proof therefore we have that $N_{j+1}^{\prime} \chi$-realizes $E^{\prime \prime}$. Let $N_{j+1}^{\prime \prime}$ be $\chi$-expansion of $N_{j+1}^{\prime}$ such that $N_{j+1}^{\prime \prime} \models E^{\prime \prime}$. By quasi-representability we lift $f_{j+1, \alpha}^{\prime}$ to $N_{j+1}^{\prime \prime} \stackrel{f_{j+1, \alpha}^{\prime \prime}}{\longrightarrow} N_{\alpha}^{\prime \prime}$ and because model homomorphisms preserve satisfaction we get that $N_{\alpha}^{\prime \prime} \models E^{\prime \prime}$. Hence $N_{\alpha}^{\prime} \chi$-realizes $E^{\prime \prime}$.

In the following we discuss the applicability of Thm. 2 by making an analysis of its underlying conditions, with special emphasis on the emblematic example of FOL. In the case of FOL the conclusion of Thm. 2 can be drawn indirectly through the respective conclusion for its more convenient technically sub-institution FOL $^{\prime}$ that restricts the signature morphisms to the injective extensions with constants and the model homomorphisms to the elementary embeddings. The same may be done for other concrete institutions too.

Condition 1. In FOL' this holds by default because all FOL'-model homomorphisms are elementary embeddings.

Condition 3. The existence of inductive colimits of signatures actually implies that we have to allow infinitely large signatures. Note that we have not imposed any finiteness condition on the signature in any of our examples. With regard to the actual condition on $\mathbf{F O L}$ ' signature morphisms, this is rather straightforward.

The inductive-exactness property on models is also a straightforward property in actual institutions, being just a special case of exactness. It is however a bit more delicate on model homomorphisms because in $\mathbf{F O L}^{\prime}$ the model homomorphisms are elementary. Luckily, we can transfer the inductive-exactness property on model homomorphisms from FOL to FOL'. Thus let us consider a chain $\left(\varphi_{i, j}\right)_{i<j \leq \lambda}$ of $\mathbf{F O L}^{\prime}$ signature morphisms and a family of FOL-model homomorphisms $\left(h_{i}\right)_{i \leq \lambda}$ such that $h_{j} \uparrow_{\varphi_{i, j}}=h_{i}$ for $i<j$. We have to establish that $h_{\lambda}$ is elementary embedding whenever $h_{i}$ is elementary embedding for each $i<\lambda$. The elementarity of $h_{\lambda}$ follows trivially because it is an expansion of any $h_{i}$ along an injective signature extension with constants.

Condition 4. Because we work within the sub-institution of the elementary embeddings, this condition is fullfilled by Tarski's Elementary Chain Theorem, which has also received an institutionindependent generalization in [25].

Condition 5. This is the single crucial condition of Thm. 2 and can be regarded as a form of compactness. For each finite $i \subseteq E$, let $A_{i}$ be the $\chi$-expansion of $A$ such that $A_{i} \mid=i$. Recall a well known compactness result in FOL stating that there exists an ultrafilter $U$ on $\mathcal{P}_{\omega}(E)$ (the set of the finite subsets of $E$ ) such that (the ultraproduct corresponding to $U$ ) $\prod_{U} A_{i} \models E$. Then $\prod_{U} A_{i} \uparrow_{\chi}=\prod_{U} A$. Also, we know that $A$ can be elementarily embedded into the ultrapower $\prod_{U} A$, which shows the condition for FOL'.

The same argument can be invoked when the role of FOL is played by any other Łoś institution ([11]; see also Dfn. 11 below) such that signature morphisms preserve filtered products.

Condition 6. This is evidently fullfilled in any institution where models consist of interpretations of the symbols of the signatures in set theoretic universes, for those signature morphisms which do not add new sorts. Note that this is obviously fullfilled by the FOL injective signature extensions with constants.

Condition 7. Since FOL' signature morphisms are injective signature extensions with constants, all signature morphisms are quasi-representable. Let $\mathcal{D}$ be the class of finitary injective signature 
extensions with constants. For each signature $\Sigma$, there exists only a set of isomorphism classes of finitary injective extensions of $\Sigma$ with constants, hence $\mathcal{D}$ is co-well-powered. For each ordinal $\lambda$, each $(\lambda, \mathcal{D})$-chain is $\lambda^{+}$-small, where $\lambda^{+}$denotes the least cardinal strictly then $\lambda$. This is a special case of the following general result, whose proof is exiled to the appendix:

Proposition 3 In any inductive-exact institution if each signature morphism of $\mathcal{D}$ is finitary and quasi-representable, then for each infinite ordinal $\lambda$, each $(\lambda, \mathcal{D})$-chain of signature morphisms is $\lambda^{+}$-small.

Based on above analysis of the conditions underlying Thm. 2 we obtain the following conventional FOL corollary together with similar corollaries for other institutions:

Corollary 1 In FOL and POA let $\mathcal{D}$ be the class of signature extensions with a finite number of constants. In $\mathbf{P A}$ let $\mathcal{D}$ be the class of the injective signature extensions with a finite number of total constants.

Then for each cardinal $\lambda$, each FOL/POA/PA model can be embedded elementarily into a $(\lambda, \mathcal{D})$-saturated model.

Although the hypotheses of the existence Thm. 2 require existential quantifiers and conjunctions, existence of saturated models can be easily extended to sub-institutions with much less expressive power of sentences.

Corollary 2 In EQL, HCL, each model can be 'elementarily embedded' into a $(\lambda, \mathcal{D})$-saturated model for each cardinal $\lambda$ and for the usual $\mathcal{D}$ consisting of the injective signature extensions with a finite number of constants.

Proof Let $M$ be a model in EQL or in HCL and consider a FOL elementary embedding $M \stackrel{h}{\rightarrow} N$ such that $N$ is $(\lambda, \mathcal{D})$-saturated in FOL. It is easy to notice that $N$ is $(\lambda, \mathcal{D})$-saturated in EQL and HCL too. Moreover $M \equiv N$ in EQL and HCL because they are elementarily equivalent in FOL. Since EQL and HCL do not make any restrictions on FOL model homomorphisms, we have that $M \stackrel{h}{\longrightarrow} N$ is a model homomorphism also in EQL and HCL, $M \equiv N$, and $N$ is $(\lambda, \mathcal{D})$-saturated.

\section{Saturated Models: uniqueness}

Definition 9 The elementary diagrams $\iota$ of an institution are simple when for each signature $\Sigma$ and all $\Sigma$-models $A, B$, for each $\iota_{\Sigma}(B)$-expansion $A^{\prime}$ of $A$, the following is a pushout square of signature morphisms.

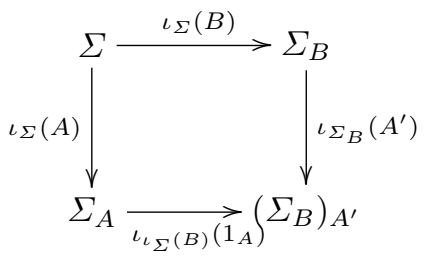

Example 10 It is easy to note that in actual examples, those elementary diagrams such that their elementary extensions just add the elements of the model as new constants to its signature, like in FOL, PA, POA etc., are simple because the above diagram is in fact a diagram of the form

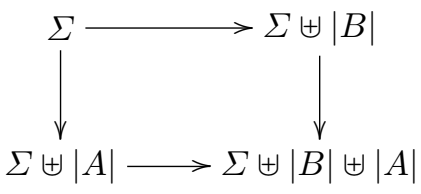


where $|A|$ and $|B|$ denote the sets of elements of (the carriers of) $A$ and $B$ and $\uplus_{-} \uplus_{-}$denotes the disjoint union of sets.

Definition 10 Let $M$ be a model in an institution with elementary diagrams $\iota$. For any cardinal number $\lambda$, we say that $M$ has $\mathcal{D}$-size $\lambda$ when $\iota_{\Sigma}(M)=\varphi_{0, \lambda}$ for some $(\lambda, \mathcal{D})$-chain $\left(\varphi_{i, j}\right)_{i<j \leq \lambda}$.

Note that the concept of 'size' introduced by Dfn. 10 above is a relation between models and cardinals rather than a function from models to cardinals.

Example 11 If we take $\mathcal{D}$ to be the class of FOL finite injective extensions of signatures with constants, a FOL model $M$ has $\mathcal{D}$-size $\operatorname{card}(|M|)$, the cardinality of its set $|M|$ of elements $\left(|M|=\cup_{s \in S} M_{s}\right.$ where $S$ is the set of the sorts of $\left.\Sigma\right)$. On the other hand, if $M$ has $\mathcal{D}$-size $\lambda$, then $\operatorname{card}(|M|) \leq \lambda$, therefore we can conclude that a model $M$ has $\mathcal{D}$-size $\lambda$ if and only if $\operatorname{card}(|M|) \leq \lambda$. By Fact 31 we can further establish the following:

Corollary 3 For any infinite cardinal $\lambda$, for each $\lambda$-saturated FOL model $M$ of size $\lambda$ such that $M_{s}$ is infinite for at least one sort, $\operatorname{card}(|M|)=\lambda$.

Theorem 3 Assume that the institution

1. has pushouts and inductive colimits of signatures,

2. is semi-exact and inductive-exact on models,

3. has simple elementary diagrams $\iota$,

4. has existential $\mathcal{D}$-quantification for a (sub)category $\mathcal{D}$ of signature morphisms which is stable under pushouts,

5. has negations and finite conjunctions, and

6. has finitary sentences.

Then any two elementary equivalent $(\lambda, \mathcal{D})$-saturated $\Sigma$-models of $\mathcal{D}$-size $\lambda$ are isomorphic.

Proof Let $M, N$ be $\Sigma$-models satisfying the hypotheses of the theorem. We consider a pushout of signature morphisms as follows:

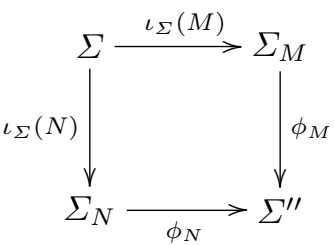

and construct elementarily equivalent $\Sigma^{\prime \prime}$-expansions, $M^{\prime \prime}$ of $M_{M}$ and $N^{\prime \prime}$ of $N_{N}$.

Suppose we have already constructed $M^{\prime \prime}$ and $N^{\prime \prime}$. Let $M^{\prime}=M^{\prime \prime} \uparrow_{\phi_{N}}$ and $N^{\prime}=N^{\prime \prime} \uparrow_{\phi_{M}}$. Because the elementary diagrams are simple and pushouts are unique up to isomorphisms, we may assume without any loss of generality that $\Sigma^{\prime \prime}=\left(\Sigma_{N}\right)_{M^{\prime}}, \phi_{M}=\iota_{\iota_{\Sigma}(N)}\left(1_{M}\right)$ and $\phi_{N}=\iota_{\Sigma_{N}}\left(M^{\prime}\right)$.

Because $M_{M^{\prime}}^{\prime} \uparrow_{\phi_{N}}=M^{\prime}$ and $M_{M^{\prime}}^{\prime} \uparrow_{\phi_{M}}=M_{M}$ (which follows from the naturality of $i$ and because $M_{M^{\prime}}^{\prime}=i_{\Sigma_{N}, M^{\prime}}^{-1}\left(1_{M^{\prime}}\right)$ and $\left.M_{M}=i_{\Sigma, M}^{-1}\left(1_{M}\right)\right)$, by the uniqueness part of the semi-exactness we get that $M^{\prime \prime}=M_{M^{\prime}}^{\prime}$.

But $N^{\prime \prime} \mid=E_{M^{\prime}}$ (because $M^{\prime \prime} \equiv N^{\prime \prime}$ ), hence we get a model homomorphism $h: M^{\prime \prime} \rightarrow N^{\prime \prime}$. Similarly we get another $\Sigma^{\prime \prime}$-homomorphism $h^{\prime}: N^{\prime \prime} \rightarrow M^{\prime \prime}$. By the initiality of $M^{\prime \prime}$ and $N^{\prime \prime}$ we have that $h ; h^{\prime}=1_{M^{\prime \prime}}$ and $h^{\prime} ; h=1_{N^{\prime \prime}}$. Thus we have that $M^{\prime \prime} \cong N^{\prime \prime}$, hence by reduction to $\Sigma$ we obtain also that $M \cong N$, which proves the theorem.

Now let us come back to the construction of $M^{\prime \prime}$ and $N^{\prime \prime}$. Since both $M$ and $N$ have the same $\mathcal{D}$ size $\lambda, \iota_{\Sigma}(M)=\varphi_{M}^{0, \lambda}$ and $\iota_{\Sigma}(N)=\varphi_{N}^{0, \lambda}$ where $\left(\Sigma_{M}^{i} \stackrel{\varphi_{M}^{i, j}}{\longrightarrow} \Sigma_{M}^{j}\right)_{i<j \leq \lambda}$ and $\left(\Sigma_{N}^{i} \stackrel{\varphi_{N}^{i, j}}{\longrightarrow} \Sigma_{N}^{j}\right)_{i<j \leq \lambda}$ are $(\lambda, \mathcal{D})$-chains of signature morphisms.

By ordinal induction we define another $(\lambda, \mathcal{D})$-chain $\left(\Sigma^{i} \stackrel{\phi^{i, j}}{\longrightarrow} \Sigma^{j}\right)_{i<j \leq \lambda}$ such that $\Sigma^{0}=\Sigma$ and for each $j<\lambda$ 


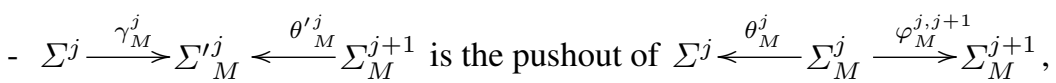

- $\Sigma^{j} \stackrel{\gamma_{N}^{j}}{\longrightarrow} \Sigma^{\prime}{ }_{N}{ }^{<}{ }_{N}^{\theta_{N}^{\prime j}} \Sigma_{N}^{j+1}$ is the pushout of $\Sigma^{j}<{ }_{N}^{\theta_{N}^{j}} \Sigma_{N}^{j} \stackrel{\varphi_{N}^{j, j+1}}{\longrightarrow} \Sigma_{N}^{j+1}$,

- $\Sigma^{\prime}{ }_{M} \stackrel{\psi_{M}^{j}}{\longrightarrow} \Sigma^{j+1} \longleftrightarrow \psi_{N}^{j} \Sigma^{\prime j}{ }_{M}$ is the pushout of $\Sigma^{\prime j}{ }_{M} \stackrel{\gamma_{M}^{j}}{\longleftarrow} \Sigma^{j} \stackrel{\gamma_{N}^{j}}{\longrightarrow} \Sigma^{\prime}{ }_{N}$,

- $\theta_{M}^{j+1}=\theta^{\prime j}{ }_{M} ; \psi_{M}^{j}$ and $\theta_{N}^{j+1}=\theta_{N}^{\prime j} ; \psi_{N}^{j}$, and

- $\phi^{j, j+1}=\gamma_{M}^{j} ; \psi_{M}^{j}=\gamma_{N}^{j} ; \psi_{N}^{j}$

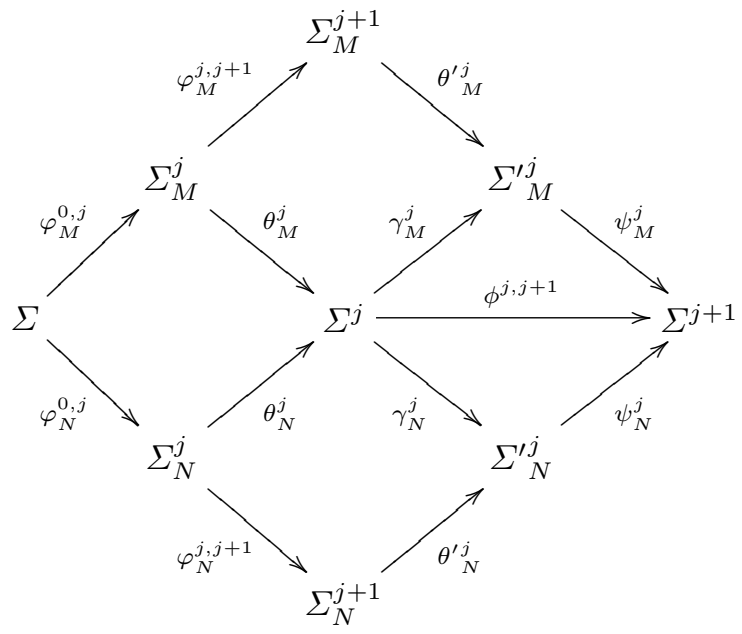

Because $\mathcal{D}$ is stable under pushouts, $\gamma_{M}^{j}, \gamma_{N}^{j}, \psi_{M}^{j}, \psi_{N}^{j} \in \mathcal{D}$, and because $\mathcal{D}$ is closed under compositions, $\phi^{j, j+1}=\gamma_{M}^{j} ; \psi_{M}^{j} \in \mathcal{D}$.

For each limit ordinal $k \leq \lambda$, we define $\Sigma_{M}^{k} \stackrel{\theta_{M}^{k}}{\longrightarrow} \Sigma^{k}$ as the unique signature morphism such that $\varphi_{M}^{i, k} ; \theta_{M}^{k}=\theta_{M}^{i} ; \phi_{M}^{i, k}$ for each $i<k . \theta_{N}^{k}$ is defined similarly. Therefore $\theta_{M}$ and $\theta_{N}$ appear as natural transformations between $\lambda$-chains, $\theta_{M}: \varphi_{M} \Rightarrow \phi$ and $\theta_{N}: \varphi_{N} \Rightarrow \phi$. Let $\phi_{M}=\theta_{M}^{\lambda}$, $\phi_{N}=\theta_{N}^{\lambda}$, and $\Sigma^{\prime \prime}=\Sigma^{\lambda}$.

It is clear from the construction that for each $j \leq \lambda$ we have that $\Sigma_{M}^{j} \stackrel{\theta_{M}^{j}}{\longrightarrow} \Sigma^{j}<{ }_{N}^{\theta_{N}^{j}} \Sigma_{N}^{j}$ is a pushout for $\Sigma_{M}^{j} \stackrel{\varphi_{M}^{0, j}}{\gtrless} \Sigma \stackrel{\varphi_{N}^{0, j}}{\longrightarrow} \Sigma_{N}^{j}$.

In the second part of the proof, by ordinal induction we define for each $j \leq \lambda, \Sigma^{j}$-models $M^{j} \equiv N^{j}$ such that $M^{j} \uparrow_{\phi^{i, j}}=M^{i}, N^{j} \uparrow_{\phi^{i, j}}=N^{i}$ for each $i \leq j$, and $M^{j} \uparrow_{\theta_{M}^{j}}=M_{M} \uparrow_{\varphi_{M}^{i, j}}$ and $N^{j} \uparrow_{\theta_{N}^{j}}=N_{N} \uparrow_{\varphi_{N}^{i, j}}$, as follows:

- $M^{0}=M$ and $N^{0}=N$,

- for each successor ordinal $j+1$ let $M^{\prime j}$ be the (unique) amalgamation $M_{M} \uparrow_{\varphi_{M}^{j+1, \lambda}} \otimes M^{j}$. For each finite $E^{\prime} \subseteq\left(M^{\prime j}\right)^{*}$, we have that $M^{j}=\left(\exists \gamma_{M}^{j}\right) \wedge E^{\prime}$ (which is a sentence of the institution because the institution has finite conjunctions and existential $\mathcal{D}$-quantification and $\left.\gamma_{M}^{j} \in \mathcal{D}\right)$. Because $M^{j} \equiv N^{j}$ we deduce that $N^{j} \models\left(\exists \gamma_{M}^{j}\right) \wedge E^{\prime}$. Because $N$ is $\lambda$-saturated, $\left(\phi^{i, k}\right)_{0 \leq i<k \leq j}$ is a $(j, \mathcal{D})$-chain with $j<\lambda, \gamma_{M}^{j} \in \mathcal{D}$, and $N^{j} \uparrow_{\phi^{0, j}}=N$, there exists a $\gamma_{M^{-}}^{j}$ expansion $N^{\prime j}$ of $N^{j}$ such that $N^{\prime j} \models\left(M^{\prime j}\right)^{*}$. Because the institution has negations, this means $N^{\prime j} \equiv M^{\prime j}$. Now we define $N^{j+1}$ to be the (unique) amalgamation $N_{N} \uparrow_{\varphi_{N}^{j+1, \lambda}} \otimes N^{\prime j}$. Like for $N^{\prime j}$, but now using the saturation of $M$, we obtain the existence of $M^{j+1} \equiv N^{j+1}$ such that $M^{j+1} \uparrow_{\psi_{M}^{j}}=M^{\prime j}$. 
- for each limit ordinal $j$, by the inductive-exactness property $M^{j}$ and $N^{j}$ are the unique $\Sigma^{j}$ models such that $\left.M^{j}\right|_{\phi^{i, j}}=M^{i}$ and $N^{j} \uparrow_{\phi^{i, j}}=N^{i}$ for each $i<j$. In order to prove $M^{j} \equiv N^{j}$, we use the fact that the institution has finitary sentences. For any $\Sigma^{j}$-sentence $\rho^{j}$ there exists $i<j$ such that $\rho^{j}=\phi^{i, j}\left(\rho^{i}\right)$ for some $\Sigma^{i}$-sentence $\rho^{i}$. Then by the Satisfaction Condition and because $M^{i} \equiv N^{i}, M^{j} \models \rho^{j}$ iff $M^{i} \models \rho^{i}$ iff $N^{i} \models \rho^{i}$ iff $N^{j} \models \rho^{j}$. That $M^{j} \uparrow_{\theta_{M}^{j}}=M_{M} \uparrow_{\varphi_{M}^{j, \lambda}}$ and $N^{j} \uparrow_{\theta_{N}^{j}}=N_{N} \uparrow_{\varphi_{N}^{j, \lambda}}$ follow by the uniqueness part of the inductiveexactness property by noticing that for each $i<j,\left(M^{j} \Gamma_{\theta_{M}^{j}}\right) \Gamma_{\varphi_{M}^{i, j}}=\left(M_{M} \Gamma_{\varphi_{M}^{j, \lambda}}\right) \Gamma_{\varphi_{M}^{i, j}}$ and $\left.\left(N^{j} \uparrow_{\theta_{N}^{j}}\right)\right|_{\varphi_{N}^{i, j}}=\left.\left(N_{N} \uparrow_{\varphi_{N}^{j, \lambda}}\right)\right|_{\varphi_{N}^{i, j}}$

Finally, $M^{\prime \prime}$ is taken as $M^{\lambda}$ and $N^{\prime \prime}$ as $N^{\lambda}$.

The following uniqueness property of saturated models is an immediate instance of the general uniqueness Thm. 3 .

Corollary 4 In FOL, PA, POA (with appropriate $\mathcal{D}$ as considered above), any two elementarily equivalent models $(\lambda, \mathcal{D})$-saturated models of cardinality $\lambda$ are isomorphic.

\section{Saturated Ultraproducts}

For this section we assume the Generalized Continuum Hypothesis,

$$
\lambda^{+}=2^{\lambda}
$$

for each infinite cardinal $\lambda$.

Let us also recall some cardinal arithmetic results needed by our work. A good reference for cardinal arithmetic is [27].

\section{Proposition 4 (Cardinal arithmetic)}

- if $\omega \leq \alpha$ then $\alpha \times \alpha=\alpha$,

- if $2 \leq \alpha \leq \beta$ and $\omega \leq \beta$ then $\alpha^{\beta}=2^{\beta}$, and

- if $\alpha \leq \beta^{+}$then $\alpha^{\beta}=\beta^{+}$.

Model ultraproducts in institutions. Let us first recall the categorical concept of ultraproduct.

Let $\mathbb{C}$ be a category with small products and directed colimits. Consider a family of objects $\left\{A_{i}\right\}_{i \in I}$. Each filter $F$ over the set of indices $I$ determines a functor $A_{F}: F \rightarrow \mathbb{C}$ such that $A_{F}\left(J \subset J^{\prime}\right)=\prod_{i \in J^{\prime}} A_{i} \stackrel{p_{J^{\prime}, J}}{\longrightarrow} \prod_{i \in J} A_{i}$ for each $J, J^{\prime} \in F$ with $J \subset J^{\prime}$, and with $p_{J^{\prime}, J}$ being the canonical projection.

Then the filtered product of $\left\{A_{i}\right\}_{i \in I}$ modulo $F$ is the colimit $\mu: A_{F} \Rightarrow \prod_{F} A_{i}$ of the functor $A_{F}$.

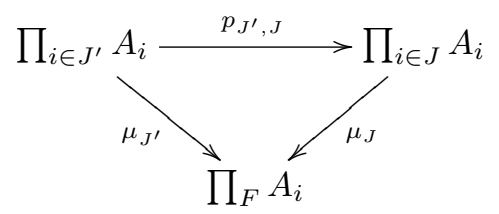

If $F$ is ultrafilter then the filtered product modulo $F$ is called an ultraproduct.

The filtered product construction from conventional model theory (in [6] called 'reduced product'; see Dfn. 4.1.6 there) has been probably defined categorically for the first time in [31] and has been used in some abstract model theoretic works, such as [2]. The equivalence between the category theoretic and the set theoretic definitions of the filtered products is shown in [23]. Filtered products of models exist in FOL, POA and PA since in all these cases the respective categories of models have all small limits and co-limits. 
Definition 11 [11] For a signature $\Sigma$ in an institution, for each filter $F \in \mathcal{F}$ over a set $I$ and for each family $\left\{A_{i}\right\}_{i \in I}$ of $\Sigma$-models, a $\Sigma$-sentence $e$ is

- preserved by $\mathcal{F}$-filtered factors if $\left.\prod_{F} A_{i}\right|_{\Sigma} e$ implies $\left\{i \in I \mid A_{i} \models_{\Sigma} e\right\} \in F$, and

- preserved by $\mathcal{F}$-filtered products if $\left\{i \in I \mid A_{i} \models_{\Sigma} e\right\} \in F$ implies $\prod_{F} A_{i} \models_{\Sigma} e$.

When $\mathcal{F}$ is the class of ultrafilters, preservation by $\mathcal{F}$-filtered factors/products is called preservation by ultrafactors/ultraproducts. A sentence is a Łos sentence when is preserved by all ultrafactors and all ultraproducts. An institution is a Łoś institution when it has all ultraproducts of models and all its sentences are Łoś sentences.

The institution-independent method of ultraproducts has been developed in [11]. The conventional Fundamental Ultraproducts Theorem shows that FOL is a Łoś institution, its institutionindependent generalization of [11] shows that a multitude of very diverse institutions are also Łoś institutions. Examples include PA, POA, EQL, HCL, etc.

Special ultrafilters. Let $(P, \leq)$ and $\left(P^{\prime}, \leq\right)$ be partial orders with binary least upper bounds $\vee$ and greatest lower bounds $\wedge$. A function $f: P \rightarrow P^{\prime}$ is

- anti-monotonic if $x<y$ implies $f(x)>f(y)$, and

- anti-additive if $f(x \vee y)=f(x) \wedge f(y)$

For any functions $f, g: P \rightarrow P^{\prime}, f \leq g$ if $f(x) \leq g(x)$ for all $x \in P$.

An ultrafilter $U$ is $\lambda$-good (see Sect. 6.1 of [6]) for a cardinal $\lambda$ if for each $\alpha<\lambda$ and each antimonotonic function $f: \mathcal{P}_{\omega}(\alpha) \rightarrow U$ there exists an anti-additive function $g: \mathcal{P}_{\omega}(\alpha) \rightarrow U$ such that $g \leq f$. (By $\mathcal{P}_{\omega}(\alpha)$ we denote the set of the finite subsets of $\alpha$ ordered by set theoretic inclusion.)

An ultrafilter $U$ over $I$ is countably incomplete if there exists an $\omega$-chain $I=I_{0} \supset I_{1} \supset \ldots \supset$ $I_{n} \supset \ldots$ such that $I_{n} \in U$ and $I_{\omega}=\bigcap_{n \in \omega} I_{n}=\emptyset$. This definition is slightly different but equivalent to that given in [6] (see Prop. 4.3.3 there).

The proof of the following theorem consists of combinatorial set-theoretic arguments, and can be found in [6] (Thm. 6.1.4).

Theorem 4 For any set I of cardinality $\lambda$, there exists $a \lambda^{+}$-good countably incomplete ultrafilter over $I$.

Definition 12 A sentence functor Sen is $\mathcal{D}$-stable for a class $\mathcal{D}$ of signature morphisms when for each $\chi: \Sigma \rightarrow \Sigma^{\prime}$ in $\mathcal{D}$ we have $\operatorname{card}\left(\operatorname{Sen}\left(\Sigma^{\prime}\right)\right) \leq \operatorname{card}(\operatorname{Sen}(\Sigma))$.

The following example is typical for a multitude of institutions, including all of those presented in this paper.

Example 12 In FOL, let $\mathcal{D}$ be the class of all injective signature extensions with a finite number of constants. We show that for each $\left(\chi: \Sigma \rightarrow \Sigma^{\prime}\right) \in \mathcal{D}$ we have that $\operatorname{card}(\operatorname{Sen}(\Sigma))=\operatorname{card}\left(\operatorname{Sen}\left(\Sigma^{\prime}\right)\right)$. Therefore $\operatorname{Sen}^{\mathbf{F O L}}$ is $\mathcal{D}$-stable.

On the one hand because $\chi$ is injective we have that $\operatorname{card}(\operatorname{Sen}(\Sigma)) \leq \operatorname{card}\left(\operatorname{Sen}\left(\Sigma^{\prime}\right)\right)$.

On the other hand the function $\operatorname{Sen}\left(\Sigma^{\prime}\right) \rightarrow \operatorname{Sen}(\Sigma)$ which maps each $\Sigma^{\prime}$-sentence $\rho^{\prime}$ to $(\exists \chi) \rho^{\prime}$ is injection, hence $\operatorname{card}\left(\operatorname{Sen}\left(\Sigma^{\prime}\right)\right) \leq \operatorname{card}(\operatorname{Sen}(\Sigma))$.

The proof of the following is given in the appendix.

Proposition 5 Consider an institution with finitary sentences and with a class $\mathcal{D}$ of signature morphisms such that the sentence functor is $\mathcal{D}$-stable. Then for each $(\alpha, \mathcal{D})$-chain $\varphi: \Sigma \rightarrow \Sigma^{\prime}$ we have that $\operatorname{card}\left(\operatorname{Sen}\left(\Sigma^{\prime}\right)\right) \leq \operatorname{card}(\alpha) \times \operatorname{card}(\operatorname{Sen}(\Sigma))$.

Theorem 5 Consider a Łoś institution with finitary sentences and with a class $\mathcal{D}$ of signature morphisms such that 
1. it has finite conjunctions and existential D-quantifications,

2. the sentence functor Sen is $\mathcal{D}$-stable,

3. the model reduct functors corresponding to $\mathcal{D}$-signature morphisms preserve ultraproducts of models, and

4. each signature morphism $\varphi: \Sigma \rightarrow \Sigma^{\prime}$ lifts completely ultraproducts, i.e. for each $\varphi$-expansion $A^{\prime}$ of an ultraproduct $\prod_{U} A_{i}$ there are $\varphi$-expansions $A_{i}^{\prime}$ of each $A_{i}$ such that $A^{\prime}=\prod_{U} A_{i}^{\prime}$.

For $\lambda$ any infinite cardinal and $U$ a countably incomplete $\lambda$-good ultrafilter over $I$, for any signature $\Sigma$, if $\operatorname{card}(\operatorname{Sen}(\Sigma))<\lambda$, then for any family $\left\{A_{i}\right\}_{i \in I}$ of $\Sigma$-models, the ultraproduct $\prod_{U} A_{i}$ is $(\lambda, \mathcal{D})$-saturated.

Proof Consider a $(\alpha, \mathcal{D})$-chain $\left(\Sigma_{i} \stackrel{\varphi_{i, j}}{\longrightarrow} \Sigma_{j}\right)_{i<j \leq \alpha}$ with $\alpha<\lambda$ such that $\Sigma_{0}=\Sigma$, a $\varphi_{0, \alpha^{-}}$ expansion $A^{\alpha}$ of $\prod_{U} A_{i},\left(\chi: \Sigma_{\alpha} \rightarrow \Sigma^{\prime}\right) \in \mathcal{D}$, and a set $E$ of $\Sigma^{\prime}$-sentences such that $A^{\alpha} \chi$-realizes $E$ finitely.

Because each signature morphism lifts completely ultraproducts, for each $i \in I$, there exists $A_{i}^{\alpha}$ $\varphi_{0, \alpha}$-expansion of $A_{i}$ such that $\prod_{U} A_{i}^{\alpha}=A_{\alpha}$.

Because $U$ is countably incomplete, there exists an $\omega$-chain $I=I_{0} \supset I_{1} \supset \ldots \supset I_{n} \supset \ldots$ such that $I_{n} \in U$ and $I_{\omega}=\bigcap_{n \in \omega} I_{n}=\emptyset$. We define $f: \mathcal{P}_{\omega}(E) \rightarrow U$ (recall that $\mathcal{P}_{\omega}(E)$ is the set of all finite subsets $E^{\prime}$ of $E$ )

- $f(\emptyset)=I$, and

- $f\left(E^{\prime}\right)=I_{n} \cap\left\{i \mid A_{i}^{\alpha} \models(\exists \chi) \wedge E^{\prime}\right\}$ where $n$ is the cardinality of $E^{\prime}$.

$f$ is well defined because $\prod_{U} A_{i}^{\alpha}=A^{\alpha} \models(\exists \chi) \wedge E^{\prime}$ and sentences are preserved by ultrafactors (hence $\left.\left\{i \in I \mid A_{i}^{\alpha} \models(\exists \chi) \wedge E^{\prime}\right\} \in U\right)$.

$f$ is also anti-monotonic because for each $E_{1} \subset E_{2} \subseteq E, I_{n_{1}}>I_{n_{2}}$ (where $n_{1}$ respectively $n_{2}$ are the cardinalities of $E_{1}$ respectively $\left.E_{2}\right)$, and $\left\{i \mid A_{i}^{\alpha} \models(\exists \chi) \wedge E_{1}\right\} \supseteq\left\{i\left|A_{i}^{\alpha}\right|=(\exists \chi) \wedge E_{2}\right\}$. Because $U$ is $\lambda$-good and the cardinality of $E$ is less than $\lambda \times \lambda=\lambda$ (see Prop. 5 and 4) there exists an anti-additive function $g: \mathcal{P}_{\omega}(E) \rightarrow U$ such that $g \leq f$. For each $i \in I$ let $E_{i}=\{\rho \in E \mid i \in$ $g(\{\rho\})\}$.

If the cardinality of $E_{i}$ is greater than $n$, then $i \in I_{n}$. In order to see this, consider $\left\{\rho_{1}, \ldots, \rho_{n}\right\} \subseteq$ $E_{i}$. This means $i \in g\left(\left\{\rho_{k}\right\}\right)$ for all $k \leq n$. As $g$ is anti-additive, we have that $i \in \bigcap_{k \leq n} g\left(\left\{\rho_{k}\right\}\right)=$ $g\left(\left\{\rho_{1}, \ldots, \rho_{n}\right\}\right) \subseteq f\left(\left\{\rho_{1}, \ldots, \rho_{n}\right\}\right) \subseteq I_{n}$.

Because $\bigcap_{n \in \omega} I_{n}=\emptyset$, for each $i \in I, E_{i}$ is finite. Otherwise if $E_{i}$ were infinite we would have that $i \in I_{n}$ for all $n \in \omega$, which contradicts $\bigcap_{n \in \omega} I_{n}=\emptyset$.

Because each $E_{i}$ is finite, we have that $i \in \bigcap_{\rho \in E_{i}} g(\{\rho\})=g\left(\bigcup_{\rho \in E_{i}}\{\rho\}\right)=g\left(E_{i}\right) \subseteq f\left(E_{i}\right)$. This means that $A_{i}^{\alpha}=(\exists \chi) \wedge E_{i}$. Let $A_{i}^{\prime}$ be the $\chi$-expansion of $A_{i}^{\alpha}$ such that $A_{i}^{\prime} \models E_{i}$.

Finally, we show that $\prod_{U} A_{i}^{\prime} \models E$. Because $\chi$ preserves ultraproducts, from $\prod_{U} A_{i}^{\prime} \models E$ we obtain that $A_{\alpha}=\prod_{U} A_{i}^{\alpha} \chi$-realizes $E$. For each $\rho \in E$, we have that $g(\{\rho\}) \subseteq\left\{i \mid A_{i}^{\prime} \models \rho\right\}$. Because $g(\{\rho\}) \in U$, we deduce that $\left\{i \mid A_{i}^{\prime} \models \rho\right\} \in U$, hence $\prod_{U} A_{i}^{\prime} \models \rho$ because $\rho$ is preserved by ultraproducts.

The conditions of Thm. 5 which need some special attention are perhaps the last two ones. The discussion can be simplified quite a lot if in actual institutions one narrows the class of the considered signature morphisms just to the injective signature extensions with constants. Therefore in FOL, the typical choice for $\mathcal{D}$ would be of course the class of all finitary signature extensions with constants. Note that this restriction on the signature morphisms does not narrow the applicability of Thm. 5, since the only signature morphisms of the institution that are involved in this result are the $(\alpha, \mathcal{D})$-chains (for $\alpha<\lambda$ ), hence the other signature morphisms apart of those mentioned above are irrelevant for this result. This situation is similar to how the existence Thm. 2 may be applied to actual situations.

Coming back to the two conditions above mentioned, the preservation of ultraproducts by the model reducts holds by the preservation of direct products and of directed colimits (cf. Prop. 1). 
Concerning the lifting condition, any interpretation $X \rightarrow \prod_{U} A_{i}$ of a set of variables in an ultraproduct of $\Sigma$-models $\prod_{U} A_{i}$ gives an interpretation $X \rightarrow \prod_{i \in I} A_{i}$ which by using the product projections further gives interpretations $X \rightarrow A_{i}$ for each $i \in I$. The interpretations $X \rightarrow A_{i}$ provides a complete lifting of $\prod_{U} A_{i}$ to an ultraproduct of $\Sigma \uplus X$-models.

Note that in some institutions Thm. 5 together with the fact that each model can be elementarily embedded in any of its ultrapowers (see [17] for a general institution-independent version of this result) may provide an alternative way to reach essentially the same conclusion as Thm. 2 . The costs are however quite high: assuming Boolean connectives, the Łoś property for the institution, and also the rather difficult result on the existence of good countably incomplete ultrafilters (Thm. 4).

The following constitutes an institution-independent generalization of the famous result proved by Keisler for FOL. (This result has been proved by Shelah without Generalized Continuum Hypothesis in [40].)

Corollary 5 Consider an institution satisfying the hypotheses of Theorems 3 and 5 and such that each model has a $\mathcal{D}$-size. Let us further assume that for each model $M$, if $M$ has a $\mathcal{D}$-size $\lambda$, then each ultrapower $\prod_{U} M$ for an ultrafilter $U$ over I has $\mathcal{D}$-size $\lambda^{\operatorname{card}(I)}$.

Then any two elementarily equivalent models have isomorphic ultrapowers (for the same ultrafilter).

Proof Let $M \equiv N$ be elementarily equivalent $\Sigma$-models. Consider a cardinal $\lambda$ such that both $M$ and $N$ have $\mathcal{D}$-size $\lambda^{+}$and such that $\operatorname{card}(\operatorname{Sen}(\Sigma)) \leq \lambda$. Let $U$ be a countably incomplete $\lambda^{+}$-good ultrafilter over $\lambda$. Then both $\prod_{U} M$ and $\prod_{U} N$ have $\mathcal{D}$-size $\left(\lambda^{+}\right)^{\lambda}=\lambda^{+}$(see Prop. 4 on cardinal arithmetic). By Thm. 5 both ultrapowers are $\left(\lambda^{+}, \mathcal{D}\right)$-saturated. By Thm. 3 they are therefore isomorphic.

Corollary 6 In FOL, PA, POA, any two elementarily equivalent models have isomorphic ultrapowers.

Proof On the one hand, in all these three institutions (with appropriate $\mathcal{D}$ defined above) hypotheses of Thm. 5 and 3 hold as discussed above. On the other hand, when we define the sizes of models by their cardinality, the specific condition of Cor. 5 holds obviously since each ultrapower $\prod_{U} M$ is the quotient of the power $\prod_{i \in I} M$.

Definition 13 An institution which has ultraproducts of models has the Keisler-Shelah property if and only if every two elementarily equivalent models have isomorphic ultrapowers.

Counterexample 51 In the sub-institution of FOL which restricts the sentences to those that do not use the equality symbol, consider the signature $\Sigma=(\{s\},\{\sigma: s \rightarrow s\}, \emptyset)$ and two models of this signature $A$ and $B$ defined as follows:

- $A_{s}=\{0,1\} ; A_{\sigma}(0)=0$ and $A_{\sigma}(1)=1$

- $B_{s}=\{0,1\} ; B_{\sigma}(0)=1$ and $B_{\sigma}(1)=0$

It is clear that $A \equiv B$ but $A$ and $B$ are not isomorphic. Because $A$ and $B$ are finite, each of them is isomorphic to any of their ultrapowers, hence for each ultrafilter $U$, ultrapowers $\prod_{U} A$ and $\prod_{U} B$ cannot be isomorphic.

This is counterexample for Keisler-Shelah property exploits an institution where the syntactic power (given by the sentences) is not enough to enforce a semantic property (isomorphism of models). The concordance between these aspects is ensured in our results by the existence of elementary diagrams. In the absence of elementary diagrams the uniqueness of saturated models (Thm. 3), which is one of the main causes for the Keisler-Shelah property, is not guaranteed.

Acknowledgements We thank Daniel Găină and Mihai Codescu for carefully studying this work and providing constructive criticism leading to correction of several mistakes and to improving our work, and Andrei Popescu for help with some cardinal theoretic issues. We are very grateful to the anonymous reviewer for a careful and competent study of our work that has resulted in a series of significant corrections. 


\section{Conclusions}

We have lifted the concept of saturated model from from conventional concrete model theory to the institution-independent model theoretic framework. We have developed the fundamental existence and uniqueness results for institution-independent saturated models. We have applied the latter result for developing a very general version of Keisler-Shelah isomorphism theorem. The former result has already been used by [8] for developing some institution-independent preservation and axiomatizability results via saturation.

Future work may develop along two rather different but related directions. One of them is to use our general theory to provide concrete saturated model theories for various less conventional logics in the same manner as we have done here about POA and PA. The other development direction is to further develop results on saturated model theory at the abstract institution-independent level of our work.

\section{References}

1. Jirí Adamek and Jirí Rosický. Locally Presentable and Accessible Categories, volume 189 of London Mathematical Society Lecture Notes. Cambridge Univ. Press, 1994.

2. Hajnal Andréka and István Németi. Łoś lemma holds in every category. Studia Scientiarum Mathematicarum Hungarica, 13:361-376, 1978

3. Edigio Astesiano, Michel Bidoit, Hélène Kirchner, Berndt Krieg-Brückner, Peter Mosses, Don Sannella, and Andrzej Tarlecki. CASL: The common algebraic specification language. Theoretical Computer Science, 286(2):153-196, 2002.

4. Michel Bidoit and Rolf Hennicker. On the integration of the observability and reachability concepts. In Proc. 5th Int. Conf. Foundations of Software Science and Computation Structures (FOSSACS'2002), volume 2303 of Lecture Notes in Computer Science, pages 21-36, 2002.

5. Tomasz Borzyszkowski. Higher-order logic and theorem proving for structured specifications. In Christine Choppy, Didier Bert, and Peter Mosses, editors, Workshop on Algebraic Development Techniques 1999, volume 1827 of LNCS, pages 401-418, 2000.

6. Chen-Chung Chang and H. Jerome Keisler. Model Theory. North Holland, Amsterdam, 1990.

7. Corina Cîrstea. An institution of modal logics for coalgebras. Logic and Algebraic Programming, 67(1-1):87-113, 2006.

8. Mihai Codescu and Daniel Găină. Preservation by saturation in institutions. Private communication.

9. Mihai Codescu and Daniel Găină. Birkhoff completeness in institutions. Logica Universalis, 2(2):277-309, 2008.

10. Răzvan Diaconescu. Extra theory morphisms for institutions: logical semantics for multi-paradigm languages. Applied Categorical Structures, 6(4):427-453, 1998. A preliminary version appeared as JAIST Technical Report IS-RR-970032F in 1997.

11. Răzvan Diaconescu. Institution-independent ultraproducts. Fundamenta Informatica, 55(3-4):321-348, 2003.

12. Răzvan Diaconescu. Elementary diagrams in institutions. Journal of Logic and Computation, 14(5):651-674, 2004.

13. Răzvan Diaconescu. An institution-independent proof of Craig Interpolation Theorem. Studia Logica, 77(1):59-79, 2004.

14. Răzvan Diaconescu. Jewels of institution-independent model theory. In Kokichi Futatsugi, José Meseguer, and JeanPierre Jouannaud, editors, Algebra, Meaning and Computation (a festschrift in honour of Professor Joseph Goguen), volume 4060 of $L N C S$, pages 65-98. Springer-Verlag Berlin Heidelberg, 2006.

15. Răzvan Diaconescu. Proof systems for institutional logic. Journal of Logic and Computation, 16(3):339-357, 2006.

16. Răzvan Diaconescu. A categorical study on the finiteness of specifications. Information Processing Letters, 108(2):75$80,2008$.

17. Răzvan Diaconescu. Institution-independent Model Theory. Birkhäuser, 2008

18. Răzvan Diaconescu and Kokichi Futatsugi. CafeOBJ Report: The Language, Proof Techniques, and Methodologies for Object-Oriented Algebraic Specification, volume 6 of AMAST Series in Computing. World Scientific, 1998.

19. Răzvan Diaconescu, Joseph Goguen, and Petros Stefaneas. Logical support for modularisation. In Gerard Huet and Gordon Plotkin, editors, Logical Environments, pages 83-130. Cambridge, 1993. Proceedings of a Workshop held in Edinburgh, Scotland, May 1991.

20. José L. Fiadeiro and José F. Costa. Mirror, mirror in my hand: A duality between specifications and models of process behaviour. Mathematical Structures in Computer Science, 6(4):353-373, 1996.

21. Joseph Goguen and Rod Burstall. Institutions: Abstract model theory for specification and programming. Journal of the Association for Computing Machinery, 39(1):95-146, 1992.

22. Joseph Goguen and Răzvan Diaconescu. Towards an algebraic semantics for the object paradigm. In Harmut Ehrig and Fernando Orejas, editors, Recent Trends in Data Type Specification, volume 785 of Lecture Notes in Computer Science, pages 1-34. Springer, 1994.

23. George Grätzer. Universal Algebra. Springer, 1979.

24. Daniel Găină and Marius Petria. Completeness by forcing. Journal of Logic and Computation, To appear. 
25. Daniel Găină and Andrei Popescu. An institution-independent generalization of Tarski's Elementary Chain Theorem. Journal of Logic and Computation, 16(6):713-735, 2006.

26. Daniel Găină and Andrei Popescu. An institution-independent proof of Robinson consistency theorem. Studia Logica, 85(1):41-73, 2007.

27. Michale Holz, Karsten Steffens, and E. Weitz. Introduction to Cardinal Arithmetic. Birkhäuser, 1999.

28. Joachim Lambek and Phil Scott. Introduction to Higher Order Categorical Logic. Cambridge, 1986. Cambridge Studies in Advanced Mathematics, Volume 7.

29. Yngve Lamo. The Institution of Multialgebras - a general framework for algebraic software development. $\mathrm{PhD}$ thesis, University of Bergen, 2003.

30. Saunders Mac Lane. Categories for the Working Mathematician. Springer, second edition, 1998.

31. Günter Matthiessen. Regular and strongly finitary structures over strongly algebroidal categories. Canadian Journal of Mathematics, 30:250-261, 1978.

32. José Meseguer. Conditional rewriting logic as a unified model of concurrency. Theoretical Computer Science, 96(1):73$155,1992$.

33. Michael Morley and Robert Vaught. Homogeneous universal models. Mathematica Scandinavica, 11:37-57, 1962.

34. Till Mossakowski. Relating CASL with other specification languages: the institution level. Theoretical Computer Science, 286:367-475, 2002.

35. Till Mossakowski, Răzvan Diaconescu, and Andrzej Tarleck. What is a logic translation? Logica Universalis, 3(1):59_ 94, 2009.

36. Till Mossakowski, Joseph Goguen, Răzvan Diaconescu, and Andrzej Tarlecki. What is a logic? In Jean-Yves Béziau, editor, Logica Universalis, pages 113-133. Birkhäuser, 2005.

37. Marius Petria and Răzvan Diaconescu. Abstract Beth definability in institutions. Journal of Symbolic Logic, 71(3):10021028, 2006.

38. Donald Sannella and Andrzej Tarlecki. Specifications in an arbitrary institution. Information and Control, 76:165-210, 1988.

39. Lutz Schröder, Till Mossakowski, and Christoph Lüth. Type class polymorphism in an institutional framework. In José Fiadeiro, editor, Recent Trends in Algebraic Development Techniques, 17th Intl. Workshop (WADT 2004), volume 3423 of Lecture Notes in Computer Science, pages 234-248. Springer, Berlin, 2004.

40. Saharon Shelah. Every two elementary equivalent models have isomorphic ultrapowers. Israel Journal of Mathematics, 10:224-233, 1971.

41. Andrzej Tarlecki. Bits and pieces of the theory of institution. In David Pitt, Samson Abramsky, Axel Poigné, and David Rydeheard, editors, Proceedings, Summer Workshop on Category Theory and Computer Programming, volume 240 of Lecture Notes in Computer Science, pages 334-360. Springer, 1986.

42. Andrzej Tarlecki. On the existence of free models in abstract algebraic institutions. Theoretical Computer Science, 37:269-304, 1986

43. Andrzej Tarlecki. Quasi-varieties in abstract algebraic institutions. Journal of Computer and System Sciences, 33(3):333-360, 1986.

44. Andrzej Tarlecki. Towards heterogeneous specifications. In D. Gabbay and M. van Rijke, editors, Proceedings, International Conference on Frontiers of Combining Systems (FroCoS'98), pages 337-360. Research Studies Press, 2000.

\section{A Exiled proofs}

This appendix contains proofs of some of the preliminary or supporting results for this work

Proposition 1. All model reduct functors corresponding to quasi-representable signature morphisms create directed colimits of models.

Proof Let $\chi: \Sigma \rightarrow \Sigma^{\prime}$ be a quasi-representable signature morphism, let $\left(A_{i}^{\prime} \stackrel{f_{i, j}^{\prime}}{\longrightarrow} A_{j}^{\prime}\right)_{(i<j) \in(I, \leq)}$ be a directed

diagram of $\Sigma^{\prime}$-models, and let $\left(A_{i} \stackrel{f_{i, j}}{\longrightarrow} A_{j}\right)_{(i<j) \in(I, \leq)}$ be its $\chi$-reduct. Consider a colimit $\left(A_{i} \stackrel{\mu_{i}}{\longrightarrow} A\right)_{i \in|I|}$ of $\left\{f_{i, j}\right\}_{(i<j) \in(I, \leq)}$. Because $\chi$ is quasi-representable, for each $i \in I$, there exists a $\chi$-expansion $\mu_{i}^{\prime}: A_{i}^{\prime} \rightarrow B_{i}$ of $\mu_{i}$. By the uniqueness property of quasi-representability and because the diagram is directed we can show that $B_{i}=B_{j}$ for all $i, j \in I$, and that $\mu_{i}^{\prime}=f_{i, j}^{\prime} ; \mu_{j}^{\prime}$ for all $(i \leq j) \in(I, \leq)$. By a similar argument we can further show that $\left\{\mu_{i}^{\prime}\right\}_{i \in I}$ is a colimit of $\left\{f_{i, j}^{\prime}\right\}_{(i<j) \in(I, \leq)}$ 


\section{Proposition 2.}

1. In any institution the (finitary) quasi-representable signature morphisms form a subcategory of $\mathbb{S} i g$.

2. If the institution is semi-exact, then quasi-representable signature morphisms are stable under pushouts

3. If the institution is directed-exact, then any directed colimit of quasi-representable signature morphisms consists of quasi-representable signature morphisms.

4. If $\varphi$ and $\varphi ; \chi$ are quasi-representable then $\chi$ is quasi-representable.

Proof 1. That composition of quasi-representable morphisms is quasi-representable follows immediately from the definition. Identity morphisms are trivially finitary quasi-representable. We focus on showing that finitary quasi-representable morphisms are closed under composition. Therefore consider $\chi: \Sigma \rightarrow \Sigma^{\prime}$ and $\chi^{\prime}: \Sigma^{\prime} \rightarrow \Sigma^{\prime \prime}$ finitary quasi-representable mor-

phisms. We have only to prove that $\chi ; \chi^{\prime}$ is finitary. Let $\left(A_{i} \stackrel{\mu_{i}}{\longrightarrow} A\right)_{i \in|I|}$ be a colimit of a directed $\operatorname{diagram}\left(A_{i} \stackrel{f_{i, j}}{\longrightarrow} A_{j}\right)_{(i<j) \in(I, \leq)}$ of $\Sigma$-model homomorphisms and let $A^{\prime \prime}$ be a $\chi ; \chi^{\prime}$-expansion of $A$. Because $\chi$ is finitary there exists $i \in|I|$ and $\mu_{i}^{\prime}: A_{i}^{\prime} \rightarrow$ $A^{\prime \prime} \Upsilon_{\chi^{\prime}}$ an $\chi$-expansion of $\mu_{i}$.

Notice that $(J, \leq)=\{j \leq k \mid i \leq j, k\}$ is also directed and is a final subcategory of $(I, \leq)$. Because $\chi$ is quasirepresentable and $(J, \leq)$ is directed, $A_{i}^{\prime}$ determines

$$
\begin{aligned}
& \text { - a } \chi \text {-expansion }\left(A_{i}^{\prime} \stackrel{f_{j, k}^{\prime}}{\longrightarrow} A_{j}^{\prime}\right)_{(j<k) \in(J, \leq)} \text { of }\left(A_{i} \stackrel{f_{j, k}}{\longrightarrow} A_{j}\right)_{(j<k) \in(J, \leq)} \text {, and } \\
& \text { - a } \text {-expansion }\left(\left.A_{j}^{\prime} \stackrel{\mu_{j}^{\prime}}{\longrightarrow} A^{\prime \prime}\right|_{\chi^{\prime}}\right)_{j \in|J|} \text { of }\left\{\mu_{j}\right\}_{j \in|J|} \text { which is a co-cone for }\left\{f_{j, k}^{\prime}\right\}_{(j<k) \in(J, \leq)} \text {. }
\end{aligned}
$$

By Prop. 1, quasi-representable signature morphisms create directed colimits, hence $\left\{\mu_{j}^{\prime}\right\}_{j \in|J|}$ is a colimiting co-cone because $\left\{\mu_{j}\right\}_{j \in|J|}$ is a colimiting co-cone (cf. Thm. 1 since $(J, \leq)$ is a final subcategory of $(I, \leq)$ ).

Now, we have only to apply the hypothesis that $\chi^{\prime}$ is finitary in order to get $j \in|J|$ and a $\chi^{\prime}$-expansion $\mu_{j}^{\prime \prime}$ of $\mu_{j}^{\prime}$. Then $\mu_{j}^{\prime \prime}$ is a $\chi ; \chi^{\prime}$-expansion of $\mu_{j}$.

2. Consider a pushout of signature morphisms

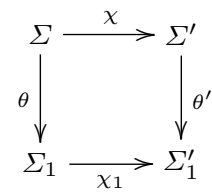

such that $\chi$ is quasi-representable. We have to show that $\chi_{1}$ is quasi-representable.

Consider a $\Sigma_{1}$-model homomorphism $h_{1}: M_{1}^{\prime} \uparrow_{\chi_{1}} \rightarrow N_{1}$. Let $h: M \rightarrow N$ be its $\theta$-reduct. $M=M_{1}^{\prime} \uparrow_{\chi_{1}} \uparrow_{\theta}=$ $M_{1}^{\prime} \uparrow_{\theta^{\prime}} \uparrow_{\chi}$. Because $\chi$ is quasi-representable, let $h^{\prime}: M_{1}^{\prime} \uparrow_{\theta^{\prime}} \rightarrow N^{\prime}$ be the unique $\chi$-expansion of $h$. By the semi-exactness of the institution, the unique amalgamation $h_{1}^{\prime}$ of $h_{1}$ and $h^{\prime}$ is the unique $\chi_{1}$-expansion from $M_{1}^{\prime}$ of $h_{1}$.

3. Let $\left(\Sigma_{i} \stackrel{\varphi_{i, j}}{\longrightarrow} \Sigma_{j}\right)_{(i<j) \in(I, \leq)}$ be a directed diagram of quasi-representable signature morphisms and let $\left(\Sigma_{i} \stackrel{\theta_{i}}{\longrightarrow} \Sigma\right)_{i \in|I|}$ be its colimit. We fix arbitrary $i \in|I|$ and show that $\theta_{i}$ is quasi-representable.

Let $M \uparrow_{\theta_{i}} \stackrel{h_{i}}{\longrightarrow} N_{i}$ be a $\Sigma_{i}$-homomorphism for some $\Sigma$-model $M$. For each $j \in I$, let $M_{j}=M \uparrow_{\theta_{j}}$. Notice that $M_{j} \uparrow \varphi_{i, j}=M_{i}$ when $j>i$. For each $j>i$, because $\varphi_{i, j}$ is quasi-representable, let $h_{j}: M_{j} \rightarrow N_{j}$ be the unique $\varphi_{i, j}$-expansion of $h_{i}$. By the uniqueness of expansion for quasi-representable signature morphisms, we can show that $h_{j^{\prime}}\left\lceil\varphi_{j, j^{\prime}}=h_{j}\right.$ for each $i \leq j<j^{\prime}$.

Now let $(J, \leq)$ be the sub-poset of $(I, \leq)$ determined by the elements $\{j \mid i \leq j\}$. Because $(J, \leq)$ is a final sub-poset of $(I, \leq)$, by Thm. 1 we deduce that $\left(\theta_{i}\right)_{i \in|J|}$ is a colimit of $\left(\varphi_{j, j^{\prime}}\right)_{\left(j<j^{\prime}\right) \in(J, \leq)}$. Because the institution is directed-exact, let $h: M \rightarrow N$ be the unique $\Sigma$-homomorphism such that $h \uparrow_{\theta_{j}}=h_{j}$ for each $j \in|J|$. Then $h$ is the unique $\theta_{i}$-expansion from $M$ of $h_{i}$

4. Let $\Sigma \stackrel{\varphi}{\longrightarrow} \Sigma^{\prime}$ and $\Sigma^{\prime} \stackrel{\chi}{\longrightarrow} \Sigma^{\prime \prime}$. Consider any $\Sigma^{\prime}$-model homomorphism $M^{\prime \prime}\left\lceil\chi \stackrel{h^{\prime}}{\longrightarrow} N^{\prime}\right.$. Then the unique $(\varphi ; \chi)$-expansion of $h^{\prime} \uparrow_{\varphi}$ to a $\Sigma^{\prime \prime}$-model homomorphism $M^{\prime \prime} \stackrel{h^{\prime \prime}}{\longrightarrow} N^{\prime \prime}$ constitutes the unique $\chi$-expansion of $h^{\prime}$ to a $\Sigma^{\prime \prime}$-model homomorphism from $M^{\prime \prime}$.

Proposition 3. In any inductive-exact institution if each signature morphism of $\mathcal{D}$ is finitary and quasi-representable, then for each infinite ordinal $\lambda$, each $(\lambda, \mathcal{D})$-chain of signature morphisms is $\lambda^{+}$-small.

Proof Consider a $(\lambda, \mathcal{D})$-chain of signature morphisms $\Sigma \stackrel{\varphi}{\longrightarrow} \Sigma^{\prime}$ and consider a $\lambda^{+}$-chain of $\Sigma$-model homomorphisms $\left(M_{i} \stackrel{h_{i, j}}{\longrightarrow} M_{j}\right)_{i<j \leq \lambda^{+}}$. Let $M_{\lambda^{+}}^{\lambda}$ be a $\varphi$-expansion of $M_{\lambda^{+}}$.

For each $0 \leq i<j \leq \lambda$, let $\Sigma_{i} \stackrel{\varphi_{i, j}}{\longrightarrow} \Sigma_{j}$ be the segment in the chain $\varphi$ determined by $i$ and $j$. 
By transfinite induction on $\alpha \leq \lambda$ we define an increasing sequence of ordinals strictly bounded by $\lambda^{+},\left\{i_{\alpha}\right\}_{\alpha \leq \lambda}$ and an inductive diagram $\left(M_{j}^{\alpha} \stackrel{h_{j, k}^{\alpha}}{\longrightarrow} M_{k}^{\alpha}\right)_{i_{\alpha} \leq j<k \leq \lambda+}$ in $\operatorname{Mod}\left(\Sigma_{\alpha}\right)$ such that $h_{j, k}^{\alpha} \uparrow_{\varphi_{\beta, \alpha}}=h_{j, k}^{\beta}$ for all $0 \leq \beta<\alpha \leq \lambda$ and $i_{\beta} \leq j<k$ as follows:

- $i_{0}=0$ and $h_{j, k}^{0}=h_{j, k}$ for all $j<k \leq \lambda^{+}$.

- Assume $\alpha=\beta+1$ is a successor ordinal. We first notice that $\left(h_{i, \lambda^{+}}^{\beta}\right)_{i_{\beta} \leq i<\lambda+}$ is a colimit of $\left(h_{j, k}^{\beta}\right)_{i_{\beta} \leq j<k<\lambda^{+}}$. This is so because $h_{j, k}^{\beta} \mid \varphi_{0, \beta}=h_{j, k},\left(h_{i, \lambda^{+}}\right)_{i_{\beta} \leq i<\lambda^{+}}$is a colimit of $\left(h_{j, k}\right)_{i_{\beta} \leq j<k<\lambda^{+}}$(since $\left(h_{j, k}\right)_{i_{\beta} \leq j<k<\lambda^{+}}$ is a final sub-diagram of $\left(h_{j, k}\right)_{0<j<k<\lambda^{+}}$; see Thm. 1), $\varphi_{0, \beta}$ is quasi-representable (by an argument similar to the argument that $\varphi$ is quasi-representable used by the proof of Thm. 2, and because quasi-representable signature morphisms create directed colimits (cf. Prop. 1)). But $M_{\lambda^{+}}^{\alpha}=M_{\lambda^{+}}^{\lambda} \Gamma_{\varphi_{\alpha, \lambda}}$ is a $\varphi_{\beta, \alpha}$-expansion of $M_{\lambda^{+}}^{\beta}$. Because $\alpha=\beta+1$, $\varphi_{\beta, \alpha} \in \mathcal{D}$, hence it is finitary. Therefore there exists $i_{\beta} \leq i_{\alpha}<\lambda^{+}$and a $\varphi_{\beta, \alpha^{2}}$-expansion $M_{i_{\alpha}}^{\alpha} \stackrel{h_{i_{\alpha}, \lambda+}^{\alpha}}{\longrightarrow} M_{\lambda^{+}}^{\alpha}$ of $h_{i_{\alpha}, \lambda^{+}}^{\beta}$. By the quasi-representability of $\varphi_{\beta, \alpha}$, by transfinite induction, this further determines an unique $\varphi_{\beta, \alpha^{-}}$ expansion $\left(h_{j, k}^{\alpha}\right)_{i_{\alpha} \leq j<k \leq \lambda^{+}}$of $\left(h_{j, k}^{\beta}\right)_{i_{\alpha} \leq j<k \leq \lambda^{+}}$.

- Assume $\alpha$ is a limit ordinal. Then we define $i_{\alpha}=\sup \left\{i_{\beta} \mid \beta<\alpha\right\}$. We have that $i_{\alpha}<\lambda^{+}$because $\alpha \leq \lambda$ and $i_{\beta}<$ $\lambda^{+}$for each $\beta<\alpha$. (This holds because one can prove that $\bigcup_{\beta<\alpha} i_{\beta}$ is ordinal and hence $i_{\alpha}=\bigcup_{\beta<\alpha} i_{\beta}$, therefore we have $\operatorname{card}\left(i_{\alpha}\right)=\operatorname{card}\left(\bigcup_{\beta<\alpha} i_{\beta}\right) \leq \operatorname{card}(\alpha) \times \operatorname{card}(\alpha) \leq \lambda \times \lambda=\lambda<\lambda^{+}$.) For all $i_{\alpha} \leq j<k \leq \lambda^{+}$ by inductive-exactness let $h_{j, k}^{\alpha}$ be the amalgamation of $\left(h_{j, k}^{\beta}\right)_{\beta<\alpha}$, i.e. the unique $\Sigma_{\alpha}$-model homomorphism such that $h_{j, k}^{\alpha}\left\lceil\varphi_{\beta, \alpha}=h_{j, k}^{\beta}\right.$.

Proposition 5 Consider an institution with finitary sentences and with a class $\mathcal{D}$ of signature morphisms such that the sentence functor is $\mathcal{D}$-stable. Then for each $(\alpha, \mathcal{D})$-chain $\varphi: \Sigma \rightarrow \Sigma^{\prime}$ we have that $\operatorname{card}\left(\operatorname{Sen}\left(\Sigma^{\prime}\right)\right) \leq \operatorname{card}(\alpha) \times$ $\operatorname{card}(\operatorname{Sen}(\Sigma))$.

Proof Let us denote the segment of the chain $\varphi$ between $i$ and $j$ by $\varphi_{i, j}: \Sigma_{i} \rightarrow \Sigma_{j}$. Then $\Sigma=\Sigma_{0}$ and $\Sigma^{\prime}=\Sigma_{\alpha}$. We prove the proposition by transfinite induction on $\alpha$.

If $\alpha$ is a successor ordinal $\beta+1$ we have that $\operatorname{card}\left(\operatorname{Sen}\left(\Sigma_{\alpha}\right)\right)=\operatorname{card}\left(\operatorname{Sen}\left(\Sigma_{\beta+1}\right)\right) \leq \operatorname{card}\left(\operatorname{Sen}\left(\Sigma_{\beta}\right)\right) \leq \operatorname{card}(\beta) \times$ $\operatorname{card}\left(\operatorname{Sen}\left(\Sigma_{0}\right)\right)=\operatorname{card}(\beta+1) \times \operatorname{card}\left(\operatorname{Sen}\left(\Sigma_{0}\right)\right)$

If $\alpha$ is a limit ordinal then $\alpha=\cup_{\beta<\alpha} \beta$. Because the institution has finitary sentences, by Lemma 1 below we obtain that $\operatorname{card}\left(\operatorname{Sen}\left(\Sigma_{\alpha}\right)\right) \leq \operatorname{card}\left(\biguplus_{\beta<\alpha} \operatorname{Sen}\left(\Sigma_{\beta}\right)\right)$. By the induction hypothesis $\operatorname{card}\left(\operatorname{Sen}\left(\Sigma_{\beta}\right)\right) \leq \operatorname{card}(\beta) \times$ $\operatorname{card}\left(\operatorname{Sen}\left(\Sigma_{0}\right)\right) \leq \operatorname{card}\left(\alpha \times \operatorname{Sen}\left(\Sigma_{0}\right)\right)$. Therefore, we have $\operatorname{card}\left(\operatorname{Sen}\left(\Sigma_{\alpha}\right)\right) \leq \operatorname{card}\left(\alpha \times \alpha \times \operatorname{Sen}\left(\Sigma_{0}\right)\right)$ and finally $\operatorname{card}\left(\operatorname{Sen}\left(\Sigma_{\alpha}\right)\right) \leq \operatorname{card}(\alpha) \times \operatorname{card}\left(\operatorname{Sen}\left(\Sigma_{0}\right)\right)$.

Lemma 1 Consider an institution with finitary sentences. Then for each limit ordinal $\alpha$ and $(\alpha, \mathcal{D})$-chain $\left(\Sigma_{i} \stackrel{\varphi_{i, j}}{\longrightarrow} \Sigma_{j}\right)_{i<j \leq \alpha}$ we have that $\operatorname{card}\left(\operatorname{Sen}\left(\Sigma_{\alpha}\right)\right) \leq \operatorname{card}\left(\biguplus_{\eta<\alpha} \operatorname{Sen}\left(\Sigma_{\beta}\right)\right)$.

Proof We define an injection $\iota$ from $\operatorname{Sen}\left(\Sigma_{\alpha}\right)$ to $\biguplus_{\beta<\alpha} \operatorname{Sen}\left(\Sigma_{\beta}\right)$. For each $\rho \in \operatorname{Sen}\left(\Sigma_{\alpha}\right)$ it exists $\Sigma_{\rho}$ a finitely presented signature, a sentence $\rho^{\text {fin }} \in \operatorname{Sen}\left(\Sigma_{\rho}\right)$ and a signature morphism $\psi_{\rho}: \Sigma_{\rho} \rightarrow \Sigma_{\alpha}$ such that $\psi_{\rho}\left(\rho^{\text {fin }}\right)=\rho$. Because $\Sigma_{\rho}$ is finitely presented and $\Sigma_{\alpha}$ is an inductive colimit there exists $\beta$ an ordinal such that $\psi_{\rho}$ factors through $\Sigma_{\beta}$. Let $\phi_{\rho}: \Sigma_{\rho} \rightarrow \Sigma_{\beta}$ such that $\phi_{\rho} ; \varphi_{\beta, \alpha}=\psi_{\rho}$. We define $\iota(\rho)$ to be $\phi_{\rho}\left(\rho^{\text {fin }}\right)$. Because $\varphi_{\beta, \alpha}(\iota(\rho))=\rho$ we get immediately that $\iota$ is an injection 